\title{
Synthesis of small carboranylsilane dendrons as scaffolds for multiple functionalization.
}

Rosario Núñez, ${ }^{\mathrm{a}}$ Arántzazu González-Campo, ${ }^{\mathrm{a}}$ Anna Laromaine, ${ }^{\mathrm{a}}$ Francesc Teixidor, ${ }^{\mathrm{a}}$ Clara Viñas, ${ }^{a^{*}}$ Reijo Sillanpää, ${ }^{b}$ Raikko Kivekäs ${ }^{\mathrm{c}}$

\section{Supporting Information}

\section{Experimental Section}

Instrumentation. IR spectra were recorded with $\mathrm{KBr}$ pellets or $\mathrm{NaCl}$ on a Shimadzu FTIR-8300 spectrophotometer. Microanalyses were performed in the analytical laboratory using a Flash EA 1112 Series microanalyzer. The electrospray-ionization mass spectra (ESI-MS) were recorded on a Bruker Esquire 3000 spectrometer using a source of ionization an ion trap analyzer. The MALDI-TOF-MS mass spectra were recorded in the negative ion mode using a Bruker Biflex MALDI-TOF $\left[\mathrm{N}_{2}\right.$ laser; $\lambda_{\text {exc }}$ $337 \mathrm{~nm}(0.5 \mathrm{~ns}$ pusles); voltage ion source $20.00 \mathrm{Kv}$ (Uis1) and $17.50 \mathrm{Kv}$ (Uis2)]. The ${ }^{1} \mathrm{H},{ }^{1} \mathrm{H}\left\{{ }^{11} \mathrm{~B}\right\} \operatorname{NMR}(300.13 \mathrm{MHz}),{ }^{13} \mathrm{C}\left\{{ }^{1} \mathrm{H}\right\}$ NMR (75.47 MHz), ${ }^{11} \mathrm{~B},{ }^{11} \mathrm{~B}\left\{{ }^{1} \mathrm{H}\right\} \mathrm{NMR}$ (96.29 MHz) and ${ }^{29} \mathrm{Si}\left\{{ }^{1} \mathrm{H}\right\}$ NMR (59.62 MHz) spectra were recorded in $\mathrm{CDCl}_{3}$ or $\left(\mathrm{CD}_{3}\right)_{2} \mathrm{CO}$ solutions on a Bruker ARX 300 spectrometer equipped with the appropriate decoupling accessories at room temperature. Chemical shift values for ${ }^{1} \mathrm{H},{ }^{1} \mathrm{H}\left\{{ }^{11} \mathrm{~B}\right\},{ }^{13} \mathrm{C}$, ${ }^{13} \mathrm{C}\left\{{ }^{1} \mathrm{H}\right\}$ NMR and ${ }^{29} \mathrm{Si}\left\{{ }^{1} \mathrm{H}\right\}$ NMR spectra were referenced to $\mathrm{SiMe}_{4}$, and those for ${ }^{11} \mathrm{~B}\left\{{ }^{1} \mathrm{H}\right\}$ and ${ }^{11} \mathrm{~B}$ NMR were referenced to external $\mathrm{BF}_{3} \cdot \mathrm{Et}_{2} \mathrm{O}$. Chemical shifts are reported in units of part per million downfield from the reference, and all coupling constants are reported in Hertz.

Material. All manipulations were carried out under an atmosphere of purified $\mathrm{N}_{2}$ using standard Schlenk techniques. Solvents were purified, dried, and distilled by standard procedures before use. 1,2- $\mathrm{C}_{2} \mathrm{~B}_{10} \mathrm{H}_{12}, 1-\mathrm{CH}_{3}-1,2-\mathrm{C}_{2} \mathrm{~B}_{10} \mathrm{H}_{11}$ and $1-\mathrm{C}_{6} \mathrm{H}_{5}-1,2-\mathrm{C}_{2} \mathrm{~B}_{10} \mathrm{H}_{11}$ were supplied by Katchem Ltd. (Prague) and used as received. $\left(\mathrm{CH}_{2}=\mathrm{CH}\right)_{3} \mathrm{SiCl}$, 
$\left(\mathrm{CH}_{3}\right)_{2} \mathrm{HSiCl}$, and Karstedt's catalyst in xylene solution (platinum divinyltetramethyldisiloxane complex, $2.1-2.4 \%$ platinum in vinyl-terminated poly(dimethylsiloxane)) and $\mathrm{I}_{2}$ were purchased from ABCR and used as received. The $n$-BuLi solution (1.6 $\mathrm{M}$ in hexanes) was used as received from Lancaster. $\mathrm{LiAlH}_{4}$, $\mathrm{C}_{6} \mathrm{H}_{5} \mathrm{CH}=\mathrm{CH}_{2}, \quad\left(\mathrm{CH}=\mathrm{CH}_{2}\right) \mathrm{C}_{6} \mathrm{H}_{4} \mathrm{CH}_{2} \mathrm{Cl}, \quad \mathrm{Mg}, \quad \mathrm{BrCH}_{2} \mathrm{CH}_{2} \mathrm{Br}$ and $\left[\mathrm{N}\left(\mathrm{CH}_{3}\right)_{4}\right] \mathrm{Cl}$ were purchased from Aldrich. The Mg was washed with hexane and THF before used.

Synthesis of 1,2-[Si(CH=CH2 $\left.)_{3}\right]_{2}-1,2-C_{2} B_{10} H_{10}$ (1). To a solution of $1,2-\mathrm{C}_{2} \mathrm{~B}_{10} \mathrm{H}_{10}$ $(1.00 \mathrm{~g}, 6.93 \mathrm{mmol})$ in $\mathrm{Et}_{2} \mathrm{O}(7 \mathrm{~mL})$ and toluene $(14 \mathrm{~mL})$ at $0{ }^{\circ} \mathrm{C}$ was added drop-wise a 1.6 M solution of $n$-BuLi in hexane $(9.1 \mathrm{~mL}, 1.46 \mathrm{mmol})$. The mixture was stirred for 1 $\mathrm{h}$ at room temperature, cooled again at $0{ }^{\circ} \mathrm{C}$, and $\left(\mathrm{CH}_{2}=\mathrm{CH}\right)_{3} \mathrm{SiCl}(1.2 \mathrm{ml}, 13.90 \mathrm{mmol})$ was added drop-wise. The reaction mixture was then stirred at room temperature for $2 \mathrm{~h}$ and filtered off through Celite. The solvent was removed in vacuo and the residue treated with cold $\mathrm{Et}_{2} \mathrm{O}(10 \mathrm{~mL})$ to isolate 1 as a white solid. Yield: $1.31 \mathrm{~g}, 52.4 \%$. A $\mathrm{Et}_{2} \mathrm{O}$ solution of $\mathbf{1}$ gave single crystals suitable for X-ray analysis. ${ }^{1} \mathrm{H}\left\{{ }^{11} \mathrm{~B}\right\}$ NMR: $\delta$ 6.27-6.17 (m, $\left.{ }^{3} \mathrm{~J}(\mathrm{H}, \mathrm{H})=16.7,{ }^{2} \mathrm{~J}(\mathrm{H}, \mathrm{H})=7.0,12 \mathrm{H}, \mathrm{CH}=\mathrm{CH}_{2}\right), 5.84\left(\mathrm{dd},{ }^{3} \mathrm{~J}(\mathrm{H}, \mathrm{H})=16.7\right.$, $\left.{ }^{2} \mathrm{~J}(\mathrm{H}, \mathrm{H})=7.0,6 \mathrm{H}, \mathrm{CH}=\mathrm{CH}_{2}\right), 2.51$ (br s, B- $\left.H\right), 2.40$ (br s, B-H), 2.21 (br s, B-H). ${ }^{11} \mathrm{~B}$ NMR: $\delta 2.5\left(\mathrm{~d},{ }^{1} \mathrm{~J}(\mathrm{~B}, \mathrm{H})=143,2 \mathrm{~B}\right),-5.5\left(\mathrm{~d},{ }^{1} \mathrm{~J}(\mathrm{~B}, \mathrm{H})=150,2 \mathrm{~B}\right),-8.7\left(\mathrm{~d},{ }^{1} \mathrm{~J}(\mathrm{~B}, \mathrm{H})=\right.$ 162, 4B), $-10.7\left(\mathrm{~d},{ }^{1} \mathrm{~J}(\mathrm{~B}, \mathrm{H})=192,2 \mathrm{~B}\right) .{ }^{13} \mathrm{C}\left\{{ }^{1} \mathrm{H}\right\}$ NMR: $\delta 138.4\left(\mathrm{CH}=\mathrm{CH}_{2}\right), 131.8$ $\left(C \mathrm{H}=\mathrm{CH}_{2}\right), 71.9\left(C_{\text {cluster }}\right) .{ }^{29} \mathrm{Si}\left\{{ }^{1} \mathrm{H}\right\}$ NMR: -18.49. FTIR $(\mathrm{KBr}), \mathrm{cm}^{-1}: 3059\left(v\left(\mathrm{C}_{\text {vinyl- }} \mathrm{H}\right)\right)$, $2574(v(\mathrm{~B}-\mathrm{H}))$. Anal. Calcd. for $\mathrm{C}_{14} \mathrm{H}_{28} \mathrm{~B}_{10} \mathrm{Si}_{2}$ : C, 46.62; H, 7.83. Found: C, 46.25; H, 7.70. MALDI-TOF-MS (m/z): cald. 360.7; found: 359.3 [M-1, $50 \%$ ], 250.1 [M$\left.\mathrm{Si}\left(\mathrm{CH}=\mathrm{CH}_{2}\right)_{3}, 15 \%\right], 143.0\left[\mathrm{M}-2\left(\mathrm{Si}\left(\mathrm{CH}=\mathrm{CH}_{2}\right)_{3}\right), 100 \%\right]$. 
Synthesis of 1-CH $-\mathrm{CH}_{3}-2-\left[\mathrm{Si}\left(\mathrm{CH}=\mathrm{CH}_{2}\right)_{3}\right]-1,2-\mathrm{C}_{2} \mathbf{B}_{10} \mathrm{H}_{10}$ (2). The procedure was the same as for compound 1 using $1.00 \mathrm{~g}(6.32 \mathrm{mmol})$ of $1-\mathrm{CH}_{3}-1,2-\mathrm{C}_{2} \mathrm{~B}_{10} \mathrm{H}_{11}$ in $\mathrm{Et}_{2} \mathrm{O}(7 \mathrm{~mL})$ and toluene $(14 \mathrm{~mL}), 4.1 \mathrm{~mL}(6.56 \mathrm{mmol})$ of $1.6 \mathrm{M}$ solution of $n$-BuLi in hexane and 1.0 $\mathrm{mL}(6.32 \mathrm{mmol})$ of $\left(\mathrm{CH}_{2}=\mathrm{CH}\right)_{3} \mathrm{SiCl}$. The reaction mixture was stirred for $50 \mathrm{~min}$ and filtered off through Celite. The solvent was removed in vacuo giving $\mathbf{2}$ as white waxy solid. Yield: $1.46 \mathrm{~g}, 86.4 \%$. A Et $2 \mathrm{O}$ solution of 2 gave single crystals suitable for $\mathrm{X}$-ray analysis. ${ }^{1} \mathrm{H}$ NMR: $\delta 6.34\left(\mathrm{dd},{ }^{3} \mathrm{~J}(\mathrm{H}, \mathrm{H})=14.6,{ }^{2} \mathrm{~J}(\mathrm{H}, \mathrm{H})=3.7,3 \mathrm{H}, \mathrm{CH}=\mathrm{CH} H_{2}\right), 6.22(\mathrm{dd}$, $\left.{ }^{3} \mathrm{~J}(\mathrm{H}, \mathrm{H})=19.5,{ }^{3} \mathrm{~J}(\mathrm{H}, \mathrm{H})=14.6,3 \mathrm{H}, \mathrm{CH}=\mathrm{CH}_{2}\right), 5.92\left(\mathrm{dd},{ }^{3} \mathrm{~J}(\mathrm{H}, \mathrm{H})=19.5,{ }^{2} \mathrm{~J}(\mathrm{H}, \mathrm{H})=3.7\right.$, $3 \mathrm{H}, \mathrm{CH}=\mathrm{CH}_{2}$ ), 3.5-1.5 (br s, B-H), $2.02\left(\mathrm{~s}, 3 \mathrm{H}, \mathrm{C}_{\text {cluster }}-\mathrm{CH}_{3}\right) .{ }^{1} \mathrm{H}\left\{{ }^{11} \mathrm{~B}\right\}$ NMR: $\delta 6.34$ (dd, $\left.{ }^{3} \mathrm{~J}(\mathrm{H}, \mathrm{H})=14.6,{ }^{2} \mathrm{~J}(\mathrm{H}, \mathrm{H})=3.7,3 \mathrm{H}, \mathrm{CH}=\mathrm{CH}_{2}\right), 6.22\left(\mathrm{dd},{ }^{3} \mathrm{~J}(\mathrm{H}, \mathrm{H})=19.5,{ }^{3} \mathrm{~J}(\mathrm{H}, \mathrm{H})=14.6\right.$, $\left.3 \mathrm{H}, \mathrm{CH}=\mathrm{CH}_{2}\right), 5.92\left(\mathrm{dd},{ }^{3} \mathrm{~J}(\mathrm{H}, \mathrm{H})=19.5,{ }^{2} \mathrm{~J}(\mathrm{H}, \mathrm{H})=3.7,3 \mathrm{H}, \mathrm{CH}=\mathrm{CH}_{2}\right), 2.95($ br s, B- $H)$, 2.45 (br s, B- $H$ ), 2.02 (s, 3H, C cluster- $\left.\mathrm{CH}_{3}\right), 1.51$ (br s, B- $\left.H\right) .{ }^{11} \mathrm{~B}$ NMR: $\delta 1.5\left(\mathrm{~d},{ }^{1} \mathrm{~J}(\mathrm{~B}, \mathrm{H})\right.$ $=130,1 \mathrm{~B}),-3.9\left(\mathrm{~d},{ }^{1} \mathrm{~J}(\mathrm{~B}, \mathrm{H})=160,1 \mathrm{~B}\right),-7.3\left(\mathrm{~d},{ }^{1} \mathrm{~J}(\mathrm{~B}, \mathrm{H})=153,4 \mathrm{~B}\right),-9.6(\mathrm{br}, 4 \mathrm{~B})$. ${ }^{13} \mathrm{C}\left\{{ }^{1} \mathrm{H}\right\}$ NMR: $\delta 138.9\left(\mathrm{CH}=C \mathrm{H}_{2}\right), 130.2\left(C \mathrm{H}=\mathrm{CH}_{2}\right), 75.2\left(C_{\text {cluster }}\right), 69.0\left(C_{\text {cluster }}\right), 25.3$ $\left(\mathrm{C}_{\text {cluster }}-\mathrm{CH}_{3}\right) .{ }^{29} \mathrm{Si}\left\{{ }^{1} \mathrm{H}\right\}$ NMR: -19.29. FTIR $(\mathrm{KBr}), \mathrm{cm}^{-1}: 3060\left(v\left(\mathrm{C}_{\text {vinyl }}-\mathrm{H}\right)\right), 2985-2950$ $\left(v\left(\mathrm{C}_{\text {alkyl }}-\mathrm{H}\right)\right), 2584(v(\mathrm{~B}-\mathrm{H}))$. Anal. Calcd. for $\mathrm{C}_{9} \mathrm{H}_{22} \mathrm{~B}_{10} \mathrm{Si}: \mathrm{C}, 40.57 ; \mathrm{H}, 8.32$. Found: $\mathrm{C}$, $40.89 ; \mathrm{H}, 8.28$.

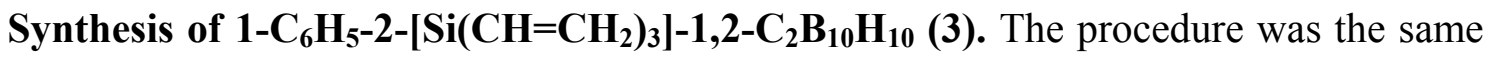
as for compound 1 using $1.01 \mathrm{~g}(4.59 \mathrm{mmol})$ of $1-\mathrm{C}_{6} \mathrm{H}_{5}-1,2-\mathrm{C}_{2} \mathrm{~B}_{10} \mathrm{H}_{11}$ in $\mathrm{Et}_{2} \mathrm{O}(7 \mathrm{~mL})$ and toluene $(14 \mathrm{~mL}), 3.0 \mathrm{~mL}(4.80 \mathrm{mmol})$ of $1.6 \mathrm{M}$ solution of $n$-BuLi in hexane and $0.8 \mathrm{~mL}(4.59 \mathrm{mmol})$ of $\left(\mathrm{CH}_{2}=\mathrm{CH}\right)_{3} \mathrm{SiCl}$. The reaction mixture was stirred for $10 \mathrm{~min}$ and filtered off through Celite. The solvent was removed in vacuo giving $\mathbf{3}$ as a white waxy solid. Yield: $1.20 \mathrm{~g}, 80.0 \%$. An $\mathrm{Et}_{2} \mathrm{O}$ solution of 3 gave single crystals suitable for X-ray analysis. ${ }^{1} \mathrm{H}$ NMR: $\delta$ 7.64-7.30 $\left(\mathrm{m}, 5 \mathrm{H}, \mathrm{C}_{6} H_{5}\right), 6.07\left(\mathrm{dd},{ }^{3} \mathrm{~J}(\mathrm{H}, \mathrm{H})=14.2\right.$, 
$\left.{ }^{2} \mathrm{~J}(\mathrm{H}, \mathrm{H})=3.2,3 \mathrm{H}, \mathrm{CH}=\mathrm{CH}_{2}\right), 5.77\left(\mathrm{dd},{ }^{3} \mathrm{~J}(\mathrm{H}, \mathrm{H})=19.9,{ }^{3} \mathrm{~J}(\mathrm{H}, \mathrm{H})=14.2,3 \mathrm{H}, \mathrm{CH}=\mathrm{CH}_{2}\right)$, $5.58\left(\mathrm{dd},{ }^{3} \mathrm{~J}(\mathrm{H}, \mathrm{H})=19.9,{ }^{2} \mathrm{~J}(\mathrm{H}, \mathrm{H})=3.2,3 \mathrm{H}, \mathrm{CH}=\mathrm{CH} H_{2}\right), 3.51-1.51$ (br s, B-H). ${ }^{1} \mathrm{H}\left\{{ }^{11} \mathrm{~B}\right\}$ NMR: $\delta$ 7.64-7.30 $\left(\mathrm{m}, 5 \mathrm{H}, \mathrm{C}_{6} H_{5}\right), 6.07\left(\mathrm{dd},{ }^{3} \mathrm{~J}(\mathrm{H}, \mathrm{H})=14.2,{ }^{2} \mathrm{~J}(\mathrm{H}, \mathrm{H})=3.2,3 \mathrm{H}\right.$, $\left.\mathrm{CH}=\mathrm{CH}_{2}\right), 5.77\left(\mathrm{dd},{ }^{3} \mathrm{~J}(\mathrm{H}, \mathrm{H})=19.9,{ }^{3} \mathrm{~J}(\mathrm{H}, \mathrm{H})=14.2,3 \mathrm{H}, \mathrm{CH}=\mathrm{CH}_{2}\right), 5.58\left(\mathrm{dd},{ }^{3} \mathrm{~J}(\mathrm{H}, \mathrm{H})=\right.$ 19.9, $\left.{ }^{2} \mathrm{~J}(\mathrm{H}, \mathrm{H})=4.1,{ }^{2} \mathrm{~J}(\mathrm{H}, \mathrm{H})=3.2,3 \mathrm{H}, \mathrm{CH}=\mathrm{CH}_{2}\right), 3.11$ (br s, B-H), 2.66 (br s, B- $H$ ), 2.19 (br s, B- $H), 1.51$ (br s, B- $H) .{ }^{11} \mathrm{~B}$ NMR: $\delta 1.5\left(\mathrm{~d},{ }^{1} \mathrm{~J}(\mathrm{~B}, \mathrm{H})=142,1 \mathrm{~B}\right),-3.7\left(\mathrm{~d},{ }^{1} \mathrm{~J}(\mathrm{~B}\right.$, $H)=145,1 B),-8.0\left(d,{ }^{1} J(B, H)=156,4 B\right),-10.1(b r, 2 B),-11.4\left(d,{ }^{1} J(B, H)=151,2 B\right)$. ${ }^{13} \mathrm{C}\left\{{ }^{1} \mathrm{H}\right\}$ NMR: $\delta 138.0\left(\mathrm{CH}=C \mathrm{H}_{2}\right), 132.3\left(\mathrm{Ph}-C_{\text {ipso }}\right), 131.5\left(\mathrm{Ph}-C_{\text {meta }}\right), 130.6\left(C \mathrm{H}=\mathrm{CH}_{2}\right)$, 129.7 (Ph- $\left.C_{\text {ortho }}\right), 128.3$ (Ph- $\left.C_{\text {para }}\right), 83.1$ ( $\left.C_{\text {cluster }}\right), 74.3$ ( $\left.C_{\text {cluster }}\right) .{ }^{29} \mathrm{Si}\left\{{ }^{1} \mathrm{H}\right\}$ NMR: -19.80. FTIR (KBr), $\mathrm{cm}^{-1}: 3060(v(\mathrm{C}-\mathrm{H})), 2574(v(\mathrm{~B}-\mathrm{H}))$. Anal. Calcd. for $\mathrm{C}_{14} \mathrm{H}_{24} \mathrm{~B}_{10} \mathrm{Si}: \mathrm{C}$, 51.18; H, 7.36. Found: C, 51.27; H 7.36.

Synthesis of 1,2-[Si( $\left.\left(\mathrm{CH}_{2} \mathrm{CH}_{2}\left(\mathrm{CH}_{3}\right)_{2} \mathrm{SiCl}\right)_{3}\right]_{2}-1,2-\mathrm{C}_{2} \mathrm{~B}_{10} \mathrm{H}_{10}$ (4). In a Schlenk flask, 1 $(0.20 \mathrm{~g}, 0.55 \mathrm{mmol}),\left(\mathrm{CH}_{3}\right)_{2} \mathrm{HSiCl}(0.8 \mathrm{~mL}, 6.59 \mathrm{mmol})$ and Karstedt catalyst $(10 \mu \mathrm{l}$, $0.02 \mathrm{mmol}$ ) were mixed and stirred for $43 \mathrm{~h}$ at room temperature. Evaporation of volatiles and the excess of $\left(\mathrm{CH}_{3}\right)_{2} \mathrm{HSiCl}$ gave 4 as a transparent oil. Yield: $0.42 \mathrm{~g}, 80.3$ \%. ${ }^{1} \mathrm{H}$ NMR: $\delta$ 3.01-2.17 (br s, B-H), 0.94 (m, 12H, Si-CH $H_{2}, 0.80$ (m, 12H, Si-CH $H_{2}$, 0.46 (s, 36H, Si-CH3). ${ }^{1} \mathrm{H}\left\{{ }^{11} \mathrm{~B}\right\} \mathrm{NMR}: \delta 2.50$ (br s, B-H), 2.27 (br s, B-H), 2.17 (br s, B$H$ ), $0.94\left(\mathrm{~m}, 12 \mathrm{H}, \mathrm{Si}-\mathrm{CH}_{2}\right), 0.80\left(\mathrm{~m}, 12 \mathrm{H}, \mathrm{Si}-\mathrm{CH}_{2}\right), 0.46$ (s, 36H, Si-CH $\left.H_{3}\right){ }^{11} \mathrm{~B}$ NMR: $\delta$ 4.1 (br, 2B), -3.7 (br, 2B), -7.7 (br, 6B). ${ }^{13} \mathrm{C}\left\{{ }^{1} \mathrm{H}\right\}$ NMR: $\delta 74.5$ (C $\left.C_{\text {cluster }}\right), 11.8\left(\mathrm{Si}-\mathrm{CH}_{2}\right)$, $6.0\left(\mathrm{Si}-\mathrm{CH}_{2}\right), 1.1\left(\mathrm{Si}-\mathrm{CH}_{3}\right) .{ }^{29} \mathrm{Si}\left\{{ }^{1} \mathrm{H}\right\}$ NMR: $32.43(\mathrm{Si}-\mathrm{Cl}), 10.75\left(\mathrm{Si}-\mathrm{C}_{\text {cluster }}\right)$.

Synthesis of 1-CH $-2-\left[\mathrm{Si}\left(\mathrm{CH}_{2} \mathrm{CH}_{2}\left(\mathrm{CH}_{3}\right)_{2} \mathrm{SiCl}\right)_{3}\right]-1,2-\mathrm{C}_{2} \mathrm{~B}_{10} \mathrm{H}_{10}$ (5). The process was the same as for compound 4 using $0.21 \mathrm{~g}(0.79 \mathrm{mmol})$ of $2,0.6 \mathrm{~mL}(5.13 \mathrm{mmol})$ of $\left(\mathrm{CH}_{3}\right)_{2} \mathrm{HSiCl}$ and $10 \mu \mathrm{l}(0.02 \mathrm{mmol})$ of Karstedt catalyst. The mixture was stirred for 16 
$\mathrm{h}$ at room temperature. Evaporation of the excess of $\left(\mathrm{CH}_{3}\right)_{2} \mathrm{HSiCl}$ gave $\mathbf{5}$ as a brown oil. Yield: 0.39 g, 90.7 \%. ${ }^{1} \mathrm{H}$ NMR: $\delta 2.04\left(\mathrm{~s}, 3 \mathrm{H}, \mathrm{C}_{\text {cluster }}-\mathrm{CH}_{3}\right), 0.91\left(\mathrm{~m}, 6 \mathrm{H}, \mathrm{Si}-\mathrm{CH}_{2}\right), 0.81$ (m, 6H, Si-CH2), 0.46 (s, 18H, Si-CH3). ${ }^{1} \mathrm{H}\left\{{ }^{11} \mathrm{~B}\right\}$ NMR: 2.44 (br s, B- $H$ ), 2.36 (br s, B$H$ ), 2.27 (br s, B-H), 2.12 (br s, B-H), 2.04 (s, 3H, C cluster- $_{\text {CH }}$ ), 0.91 (m, 6H, Si-CH2), $0.81\left(\mathrm{~m}, 6 \mathrm{H}, \mathrm{Si}-\mathrm{CH}_{2}\right), 0.46\left(\mathrm{~s}, 18 \mathrm{H}, \mathrm{Si}-\mathrm{CH}_{3}\right) .{ }^{11} \mathrm{~B} \mathrm{NMR}: \delta 2.6\left(\mathrm{~d},{ }^{1} \mathrm{~J}(\mathrm{~B}, \mathrm{H})=106,1 \mathrm{~B}\right),-$ $3.4\left(\mathrm{~d},{ }^{1} \mathrm{~J}(\mathrm{~B}, \mathrm{H})=140,1 \mathrm{~B}\right),-6.4\left(\mathrm{~d},{ }^{1} \mathrm{~J}(\mathrm{~B}, \mathrm{H})=106,6 \mathrm{~B}\right),-8.7(\mathrm{br}, 2 \mathrm{~B}) .{ }^{13} \mathrm{C}\left\{{ }^{1} \mathrm{H}\right\} \mathrm{NMR}: \delta$ $75.9\left(C_{\text {cluster }}\right), 70.8\left(C_{\text {cluster }}\right), 26.2\left(\mathrm{C}_{\text {cluster }}-C_{3}\right), 11.3\left(\mathrm{Si}-\mathrm{CH}_{2}\right), 4.58\left(\mathrm{Si}-\mathrm{CH}_{2}\right), 0.99$ (Si$\left.\mathrm{CH}_{3}\right) .{ }^{29} \mathrm{Si}\left\{{ }^{1} \mathrm{H}\right\}$ NMR: $32.24(\mathrm{Si}-\mathrm{Cl}), 9.45\left(\mathrm{Si}-\mathrm{C}_{\text {cluster }}\right)$.

Synthesis of 1- $\mathrm{C}_{6} \mathrm{H}_{5}-2-\left[\mathrm{Si}\left(\mathrm{CH}_{2} \mathrm{CH}_{2}\left(\mathrm{CH}_{3}\right)_{2} \mathrm{SiCl}\right)_{3}\right]-1,2-\mathrm{C}_{2} \mathrm{~B}_{10} \mathrm{H}_{10}$ (6): The process was the same as for compound 4 using $0.21 \mathrm{~g}(0.64 \mathrm{mmol})$ of $3,0.5 \mathrm{~mL}(4.28 \mathrm{mmol})$ of $\mathrm{HSiMe}_{2} \mathrm{Cl}$ and $10 \mu \mathrm{l}(0.02 \mathrm{mmol})$ of Karstedt catalyst. The mixture was stirred for $14 \mathrm{~h}$ at room temperature. Evaporation of the excess of $\left(\mathrm{CH}_{3}\right)_{2} \mathrm{HSiCl}$ gave $\mathbf{6}$ as a yellow waxy solid. Yield: $0.37 \mathrm{~g}, 94.9 \% .{ }^{1} \mathrm{H}$ NMR: $\delta$ 7.69-7.33 (m, 5H, $\left.\mathrm{C}_{6} H_{5}\right), 0.60(\mathrm{~m}, 6 \mathrm{H}$, Si-CH $\left.H_{2}\right), 0.49\left(\mathrm{~m}, 6 \mathrm{H}, \mathrm{Si}-\mathrm{CH}_{2}\right), 0.39$ (s, 18H, Si-CH$) .{ }^{1} \mathrm{H}\left\{{ }^{11} \mathrm{~B}\right\}$ NMR: $\delta$ 7.69-7.33 (m, 5H, $\mathrm{C}_{6} H_{5}$ ), 2.94 (br s, B-H), 2.60 (br s, B- $H$ ), 2.40 (br s, B- $H$ ), 2.25 (br s, B- $H$ ), 0.60 (m, 6H, Si-CH $\left.H_{2}\right), 0.49\left(\mathrm{~m}, 6 \mathrm{H}, \mathrm{Si}-\mathrm{CH}_{2}\right), 0.39\left(\mathrm{~s}, 18 \mathrm{H}, \mathrm{Si}-\mathrm{CH}_{3}\right) .{ }^{11} \mathrm{~B}$ NMR: $\delta 3.6\left(\mathrm{~d},{ }^{1} \mathrm{~J}(\mathrm{~B}, \mathrm{H})\right.$ $=113,1 \mathrm{~B}),-1.3\left(\mathrm{~d},{ }^{1} \mathrm{~J}(\mathrm{~B}, \mathrm{H})=145,1 \mathrm{~B}\right),-6.3\left(\mathrm{~d},{ }^{1} \mathrm{~J}(\mathrm{~B}, \mathrm{H})=147,4 \mathrm{~B}\right),-8.8(\mathrm{br}, 2 \mathrm{~B}),-$ 10.07 (br, 2B). ${ }^{13} \mathrm{C}\left\{{ }^{1} \mathrm{H}\right\}$ NMR: $\delta 132.8$ (Ph- $\left.C_{\text {ipso }}\right), 131.3$ (Ph- $\left.C_{\text {meta }}\right), 130.8$ (Ph- $\left.C_{\text {ortho }}\right)$, $128.8\left(\mathrm{Ph}-C_{\text {para }}\right), 84.0\left(C_{\text {cluster }}\right), 76.2\left(C_{\text {cluster }}\right), 11.2\left(\mathrm{Si}-C_{2}\right), 3.8\left(\mathrm{Si}-C_{2} \mathrm{H}_{2}\right), 1.0\left(\mathrm{Si}-C_{3}\right)$. ${ }^{29} \mathrm{Si}\left\{{ }^{1} \mathrm{H}\right\}$ NMR: 32.20 (Si-Cl), $10.28($ Si-C cluster $)$.

Synthesis of 1,2-[Si(C $\left.\left(\mathrm{CH}_{2} \mathrm{CH}_{2}\left(\mathrm{CH}_{3}\right)_{2} \mathrm{SiH}\right)_{3}\right]_{2}-1,2-\mathrm{C}_{2} \mathrm{~B}_{10} \mathrm{H}_{10}$ (7): To a solution of $\mathrm{LiAlH}_{4}$ (64.1 $\mathrm{mg}, 1.69 \mathrm{mmol})$ in $\mathrm{Et}_{2} \mathrm{O}(10 \mathrm{~mL})$ at $0{ }^{\circ} \mathrm{C}$ was added drop-wise a solution of 4 $(0.52 \mathrm{~g}, 0.56 \mathrm{mmol})$ in $\mathrm{Et}_{2} \mathrm{O}(10 \mathrm{~mL})$. The mixture was stirred for $15 \mathrm{~h}$ at room 
temperature and filtered off through Celite twice. The solvent was removed in vacuo to give 7 as a transparent oil. Yield: $0.24,58.5 \% .{ }^{1} \mathrm{H}$ NMR: $\delta 3.87(\mathrm{~m}, 6 \mathrm{H}, \mathrm{Si}-H), 3.01-$ 2.17 (br s, B-H), $0.83\left(\mathrm{~m}, 12 \mathrm{H}, \mathrm{Si}-\mathrm{CH}_{2}\right), 0.57$ (m, 12H, Si-CH2), $0.12\left(\mathrm{~d},{ }^{3} \mathrm{~J}(\mathrm{H}, \mathrm{H})=3.7\right.$, 36H, Si-C $\left.H_{3}\right) .{ }^{1} \mathrm{H}\left\{{ }^{11} \mathrm{~B}\right\}$ NMR: $\delta 3.87$ (m, 6H, Si-H), 2.47 (br s, B- $H$ ), 2.31 (br s, B- $H$ ), 2.17 (br s, B-H), 0.83 (m, 12H, Si-CH $), 0.57$ (m, 12H, Si-CH $), 0.12\left(\mathrm{~d},{ }^{3} \mathrm{~J}(\mathrm{H}, \mathrm{H})=3.7\right.$, 36H, Si-CH3). ${ }^{11}$ B NMR: $\delta 3.6$ (br, 2B), -3.3 (br, 2B), -7.7 (br, 6B). ${ }^{13} \mathrm{C}\left\{{ }^{1} \mathrm{H}\right\}$ NMR: $\delta$ $75.7\left(C_{\text {cluster }}\right), 6.6\left(\mathrm{Si}-\mathrm{CH}_{2}\right), 6.1\left(\mathrm{Si}-\mathrm{CH}_{2}\right),-4.9\left(\mathrm{Si}-\mathrm{CH}_{3}\right) .{ }^{29} \mathrm{Si}\left\{{ }^{1} \mathrm{H}\right\} \quad \mathrm{NMR}: 9.60$ (Si$\left.\mathrm{C}_{\text {cluster }}\right),-9.62(\mathrm{Si}-\mathrm{H})$. FTIR $(\mathrm{NaCl}), \mathrm{cm}^{-1}: 2957-2900\left(v\left(\mathrm{C}_{\text {alkyl-H}}\right)\right), 2584(v(\mathrm{~B}-\mathrm{H})), 2112$ $(v(\mathrm{Si}-\mathrm{H})), 1249\left(\delta\left(\mathrm{Si}_{-} \mathrm{CH}_{3}\right)\right)$.

Synthesis of 1-CH$-2-\left[\mathrm{Si}\left(\mathrm{CH}_{2} \mathrm{CH}_{2}\left(\mathrm{CH}_{3}\right)_{2} \mathrm{SiH}\right)_{3}\right]-1,2-\mathrm{C}_{2} \mathrm{~B}_{10} \mathrm{H}_{10}$ (8): The process was the same as for compound 7 using $\mathrm{LiAlH}(40.1 \mathrm{mg}, 1.00 \mathrm{mmol})$ of in $\mathrm{Et}_{2} \mathrm{O}(10 \mathrm{~mL})$ and $5\left(0.37(0.67 \mathrm{mmol})\right.$ in $\mathrm{Et}_{2} \mathrm{O}(5 \mathrm{~mL})$. The mixture was stirred for $15 \mathrm{~h}$ at room temperature and filtered off through Celite three times. The solvent was removed in vacuo to give 8 as a transparent oil. Yield: $0.18 \mathrm{~g}, 60.0 \% .{ }^{1} \mathrm{H}$ NMR: $\delta 3.87\left(\mathrm{sept},{ }^{3} \mathrm{~J}(\mathrm{H}\right.$, $\mathrm{H})=3.7,3 \mathrm{H}, \mathrm{Si}-\mathrm{H}), 2.03\left(\mathrm{~s}, 3 \mathrm{H}, \mathrm{C}_{\text {cluster }}-\mathrm{CH}_{3}\right), 0.84\left(\mathrm{~m}, 6 \mathrm{H}, \mathrm{Si}-\mathrm{CH}_{2}\right), 0.58(\mathrm{~m}, 6 \mathrm{H}, \mathrm{Si}-$ $\left.\mathrm{CH}_{2}\right), 0.13\left(\mathrm{~d},{ }^{3} \mathrm{~J}(\mathrm{H}, \mathrm{H})=3.7,18 \mathrm{H}, \mathrm{Si}-\mathrm{CH} H_{3}\right) .{ }^{1} \mathrm{H}\left\{{ }^{11} \mathrm{~B}\right\}$ NMR: $3.87\left(\operatorname{sept},{ }^{3} \mathrm{~J}(\mathrm{H}, \mathrm{H})=3.7\right.$, 3H, Si-H), 2.42 (br s, B-H), 2.36 (br s, B-H), 2.26 (br s, B-H), 2.16 (br s, B-H), 2.03 (s, $\left.3 \mathrm{H}, \mathrm{C}_{\text {cluster- }}-\mathrm{CH}_{3}\right), 0.84\left(\mathrm{~m}, 6 \mathrm{H}, \mathrm{Si}-\mathrm{CH}_{2}\right), 0.58\left(\mathrm{~m}, 6 \mathrm{H}, \mathrm{Si}-\mathrm{CH}_{2}\right), 0.13\left(\mathrm{~d},{ }^{3} \mathrm{~J}(\mathrm{H}, \mathrm{H})=3.7\right.$, 18H, Si-CH 3$).{ }^{11}$ B NMR: $\delta 2.2\left(\mathrm{~d},{ }^{1} \mathrm{~J}(\mathrm{~B}, \mathrm{H})=133,1 \mathrm{~B}\right),-3.4\left(\mathrm{~d},{ }^{1} \mathrm{~J}(\mathrm{~B}, \mathrm{H})=158,1 \mathrm{~B}\right),-6.5$ $\left(\mathrm{d},{ }^{1} \mathrm{~J}(\mathrm{~B}, \mathrm{H})=151,6 \mathrm{~B}\right),-8.5(\mathrm{br}, 2 \mathrm{~B}) .{ }^{13} \mathrm{C}\left\{{ }^{1} \mathrm{H}\right\}$ NMR: $75.7\left(C_{\text {cluster }}\right), 71.8\left(C_{\text {cluster }}\right), 26.1$ $\left(\mathrm{C}_{\text {cluster }}-\mathrm{CH}_{3}\right), 6.6\left(\mathrm{Si}-\mathrm{CH}_{2}\right), 6.1\left(\mathrm{Si}-\mathrm{CH}_{2}\right),-4.9\left(\mathrm{Si}-\mathrm{CH}_{3}\right) .{ }^{29} \mathrm{Si}\left\{{ }^{1} \mathrm{H}\right\} \quad \mathrm{NMR}: 9.30$ (Si$\left.\mathrm{C}_{\text {cluster }}\right),-9.82(\mathrm{Si}-\mathrm{H})$. FTIR $(\mathrm{NaCl}), \mathrm{cm}^{-1}: 2957-2900\left(v\left(\mathrm{C}_{\text {alkyl }}-\mathrm{H}\right)\right), 2584(v(\mathrm{~B}-\mathrm{H})), 2112$ $(v(\mathrm{Si}-\mathrm{H})), 1249\left(\delta\left(\mathrm{Si}_{-} \mathrm{CH}_{3}\right)\right)$. MALDI-TOF-MS $(\mathrm{m} / \mathrm{z}): 445.31$ [M-2, $20 \%$ ], 155.98 [M$\left.\left(\mathrm{C}_{12} \mathrm{H}_{33} \mathrm{Si}_{4}\right), 100 \%\right]$. 
Synthesis of 1- $\mathrm{C}_{6} \mathrm{H}_{5}-2-\left[\mathrm{Si}\left(\mathrm{CH}_{2} \mathrm{CH}_{2} \mathrm{Si}\left(\mathrm{CH}_{3}\right)_{2} \mathrm{H}\right)_{3}\right]-1,2-\mathrm{C}_{2} \mathrm{~B}_{10} \mathrm{H}_{10}$ (9): The process was

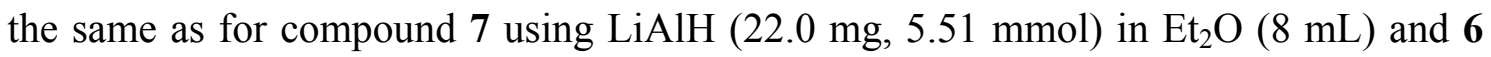
(0.22 $\mathrm{g}, 0.36 \mathrm{mmol})$ in $\mathrm{Et}_{2} \mathrm{O}(5 \mathrm{~mL})$. The mixture was stirred for $16 \mathrm{~h}$ at room temperature and filtered off through Celite three times. The solvent was removed in vacuo giving 9 as a transparent oil. Yield: $0.15 \mathrm{~g}, 78.9 \% .{ }^{1} \mathrm{H}$ NMR: $\delta 7.69-7.34(\mathrm{~m}, 5 \mathrm{H}$, $\left.\mathrm{C}_{6} H_{5}\right), 3.77\left(\mathrm{sept},{ }^{3} \mathrm{~J}(\mathrm{H}, \mathrm{H})=3.7,3 \mathrm{H}, \mathrm{Si}-H\right), 0.40$ (br s, $\left.12 \mathrm{H}, \mathrm{Si}-\mathrm{CH}_{2}\right), 0.05\left(\mathrm{~d},{ }^{3} \mathrm{~J}(\mathrm{H}, \mathrm{H})\right.$ $\left.=3.7,18 \mathrm{H}, \mathrm{Si}-\mathrm{C} H_{3}\right) .{ }^{1} \mathrm{H}\left\{{ }^{11} \mathrm{~B}\right\} \mathrm{NMR}: \delta$ 7.69-7.34 (m, 5H, $\left.\mathrm{C}_{6} H_{5}\right), 3.77\left(\mathrm{sept},{ }^{3} \mathrm{~J}(\mathrm{H}, \mathrm{H})=\right.$ 3.7, 3H, Si-H), 2.93 (br s, B-H), 2.57 (br s, B-H), 2.38 (br s, B- $H$ ), 2.26 (br s, B- $H$ ), 0.40 (br s, $\left.12 \mathrm{H}, \mathrm{Si}-\mathrm{CH}_{2}\right), 0.05\left(\mathrm{~d},{ }^{3} \mathrm{~J}(\mathrm{H}, \mathrm{H})=3.7,18 \mathrm{H}, \mathrm{Si}-\mathrm{CH}_{3}\right) .{ }^{11} \mathrm{~B} \mathrm{NMR}: \delta 3.0\left(\mathrm{~d},{ }^{1} \mathrm{~J}(\mathrm{~B}, \mathrm{H})\right.$ $=118,1 \mathrm{~B}),-1.5\left(\mathrm{~d},{ }^{1} \mathrm{~J}(\mathrm{~B}, \mathrm{H})=144,1 \mathrm{~B}\right),-6.4\left(\mathrm{~d},{ }^{1} \mathrm{~J}(\mathrm{~B}, \mathrm{H})=153,4 \mathrm{~B}\right),-8.5(\mathrm{br}, 2 \mathrm{~B}),-10.2$ (br, 2B). ${ }^{13} \mathrm{C}\left\{{ }^{1} \mathrm{H}\right\}$ NMR: $\delta 133.1$ (Ph- $\left.C_{\text {ipso }}\right), 131.3$ (Ph- $\left.C_{\text {meta }}\right), 130.5$ (Ph- $\left.C_{\text {ortho }}\right), 128.5$ $\left(\mathrm{Ph}-C_{\text {para }}\right), 83.9\left(C_{\text {cluster }}\right), 6.4\left(\mathrm{Si}-C_{2}\right), 5.3\left(\mathrm{Si}-\mathrm{CH}_{2}\right),-4.9\left(\mathrm{Si}-\mathrm{CH}_{3}\right) .{ }^{29} \mathrm{Si}\left\{{ }^{1} \mathrm{H}\right\}$ NMR: 10.27 (Si-C $\left.\mathrm{C}_{\text {cluster }}\right),-10.04(\mathrm{Si}-\mathrm{H})$. FTIR $(\mathrm{NaCl}), \mathrm{cm}^{-1}: 3064\left(\mathrm{v}\left(\mathrm{C}_{\mathrm{aryl}}-\mathrm{H}\right)\right), 2957-2903$ $\left(v\left(\mathrm{C}_{\text {alkyl-H}}\right)\right), 2586(v(\mathrm{~B}-\mathrm{H})), 2110(v(\mathrm{Si}-\mathrm{H})), 1249\left(\delta\left(\mathrm{Si}_{-} \mathrm{CH}_{3}\right)\right)$. MALDI-TOF-MS $(\mathrm{m} / \mathrm{z})$ : $508.41[\mathrm{M}-1,100 \%], 218.07\left[\mathrm{C}_{8} \mathrm{~B}_{10} \mathrm{H}_{14}, 50 \%\right]$.

Synthesis of 1-CH $3-2-\left[\mathrm{Si}\left(\mathrm{CH}_{2} \mathrm{CH}_{2} \mathrm{Si}\left(\mathrm{CH}_{3}\right)_{2} \mathrm{CH}_{2} \mathrm{CH}_{2} \mathrm{C}_{6} \mathrm{H}_{5}\right)_{3}\right]-1,2-\mathrm{C}_{2} \mathrm{~B}_{10} \mathrm{H}_{10}$ (10): In a Schlenk flask, 8 (0.11 g, $0.24 \mathrm{mmol}), \mathrm{C}_{6} \mathrm{H}_{5} \mathrm{CH}=\mathrm{CH}_{2}(8.3 \mu \mathrm{L}, 0.72 \mathrm{mmol})$ and Karstedt catalyst $(5.0 \mu \mathrm{L}, 0.01 \mathrm{mmol})$ were mixed and stirred overnight at room temperature. The volatiles were removed in vacuo giving $\mathbf{1 0}$ as a yellow oil. Yield: $0.18 \mathrm{~g}, \sim 99 \% .{ }^{1} \mathrm{H}$ NMR: $\delta$ 7.33-7.21 (m, 15H, $\left.\mathrm{C}_{6} H_{5}\right), 2.67\left(\mathrm{t},{ }^{3} \mathrm{~J}(\mathrm{H}, \mathrm{H})=8.5,6 \mathrm{H}, \mathrm{CH}_{2}-\mathrm{C}_{6} \mathrm{H}_{5}\right), 2.01(\mathrm{~s}, 3 \mathrm{H}$, $\left.\mathrm{C}_{\text {cluster }}-\mathrm{CH}_{3}\right), 0.95\left(\mathrm{t},{ }^{3} \mathrm{~J}(\mathrm{H}, \mathrm{H})=8.5,6 \mathrm{H}, \mathrm{Si}-\mathrm{CH}_{2}\right), 0.74\left(\mathrm{~m}, 6 \mathrm{H}, \mathrm{Si}-\mathrm{CH}_{2}\right), 0.47(\mathrm{~m}, 6 \mathrm{H}$, Si-C $\left.H_{2}\right), 0.06\left(\mathrm{~s}, 18 \mathrm{H}, \mathrm{Si}-\mathrm{CH}_{3}\right) .{ }^{1} \mathrm{H}\left\{{ }^{11} \mathrm{~B}\right\}$ NMR: $\delta$ 7.33-7.21 (m, 15H, $\left.\mathrm{C}_{6} H_{5}\right), 2.67(\mathrm{t}$, $\left.{ }^{3} \mathrm{~J}(\mathrm{H}, \mathrm{H})=8.5,6 \mathrm{H}, \mathrm{CH}_{2}-\mathrm{C}_{6} \mathrm{H}_{5}\right), 2.49$ (br, B-H), 2.35 (br s, B-H), 2.29 (br s, B-H), 2.19 
(br s, B-H), $2.01\left(\mathrm{~s}, 3 \mathrm{H}, \mathrm{C}_{\text {cluster }}-\mathrm{CH}_{3}\right), 0.95\left(\mathrm{t},{ }^{3} \mathrm{~J}(\mathrm{H}, \mathrm{H})=8.5,6 \mathrm{H}, \mathrm{Si}-\mathrm{CH}_{2}\right), 0.74(\mathrm{~m}, 6 \mathrm{H}$, Si-C $\left.H_{2}\right), 0.47\left(\mathrm{~m}, 6 \mathrm{H}, \mathrm{Si}-\mathrm{CH}_{2}\right), 0.06\left(\mathrm{~s}, 18 \mathrm{H}, \mathrm{Si}-\mathrm{CH}_{3}\right) .{ }^{11} \mathrm{~B}$ NMR: $\delta 2.2$ (br, 1B), -3.4 (br, 1B), -6.5 (br, 8B). ${ }^{13} \mathrm{C}\left\{{ }^{1} \mathrm{H}\right\}$ NMR: $\delta$ 144.8, 128.3, 127.7, $125.7\left(C_{6} \mathrm{H}_{5}\right), 75.7$ $\left(C_{\text {cluster }}\right), 72.1\left(C_{\text {cluster }}\right), 30.0\left(\mathrm{CH}_{2}-\mathrm{C}_{6} \mathrm{H}_{5}\right), 26.2\left(\mathrm{C}_{\text {cluster }}-\mathrm{CH}_{3}\right), 16.6\left(\mathrm{Si}-\mathrm{CH}_{2}\right), 7.6$ (Si$\left.\mathrm{CH}_{2}\right), 5.3\left(\mathrm{Si}-\mathrm{CH}_{2}\right),-4.0\left(\mathrm{Si}-\mathrm{CH}_{3}\right) .{ }^{29} \mathrm{Si}\left\{{ }^{1} \mathrm{H}\right\} \mathrm{NMR}: 9.37\left(\mathrm{Si}-\mathrm{C}_{\text {cluster }}\right), 4.73\left(\mathrm{Si}-\mathrm{CH}_{2}\right)$. FTIR $(\mathrm{NaCl}), \mathrm{cm}^{-1}: 3062\left(v\left(\mathrm{C}_{\text {aryl }}-\mathrm{H}\right)\right), 2921\left(v\left(\mathrm{C}_{\text {alkyl }}-\mathrm{H}\right)\right), 2582(v(\mathrm{~B}-\mathrm{H})), 1249(\delta(\mathrm{Si}-$ $\mathrm{CH}_{3}$ )). Anal. Calcd for $\mathrm{C}_{39} \mathrm{H}_{70} \mathrm{~B}_{10} \mathrm{Si}_{4}: \mathrm{C}, 61.68 ; \mathrm{H}, 9.29 \%$. Found: C, 61.20; H, 9.21. MSElectrospray (ESI) solution of $\mathrm{CHCl}_{3} / \mathrm{MeOH} \mathrm{m} / \mathrm{z}$ calcd for $\mathrm{C}_{39} \mathrm{H}_{70} \mathrm{~B}_{10} \mathrm{Si}_{4}$ : 759.4 ; found: $782.5\left[(\mathrm{M}+\mathrm{Na})^{+}, 12 \%\right]$.

\section{Synthesis of $1-\mathrm{C}_{6} \mathrm{H}_{5}-2-\left[\mathrm{Si}\left(\mathrm{CH}_{2} \mathrm{CH}_{2}\left(\mathrm{CH}_{3}\right)_{2} \mathrm{SiCH}_{2} \mathrm{CH}_{2} \mathrm{C}_{6} \mathrm{H}_{4} \mathrm{CH}_{2} \mathrm{Cl}\right)_{3}\right]-1,2-\mathrm{C}_{2} \mathrm{~B}_{10} \mathrm{H}_{10}$} (11). In a Schlenk flask, $9(0.15 \mathrm{~g}, 0.29 \mathrm{mmol}),\left(\mathrm{CH}=\mathrm{CH}_{2}\right) \mathrm{C}_{6} \mathrm{H}_{4} \mathrm{CH}_{2} \mathrm{Cl}(0.13 \mathrm{~mL}, 0.86$ mmol) and Karstedt catalyst $(5 \mu 1,0.01 \mathrm{mmol})$ were mixed and stirred overnight at room temperature. The volatiles were removed in vacuo to give $\mathbf{1 1}$ as a yellow oil. Yield: $0.28 \mathrm{~g}, \sim 99 \% .{ }^{1} \mathrm{H}$ NMR: $\delta$ 7.69-7.34 (m, 5H, $\left.\mathrm{C}_{6} H_{5}\right), 7.33\left(\mathrm{~d},{ }^{3} \mathrm{~J}(\mathrm{H}, \mathrm{H})=8.5,6 \mathrm{H}\right.$, $\left.\mathrm{C}_{6} \mathrm{H}_{4}\right), 7.17\left(\mathrm{~d},{ }^{3} \mathrm{~J}(\mathrm{H}, \mathrm{H})=4.1,6 \mathrm{H}, \mathrm{C}_{6} H_{4}\right), 4.59\left(\mathrm{~s}, 6 \mathrm{H}, \mathrm{CH}_{2}-\mathrm{Cl}\right), 2.58\left(\mathrm{tt},{ }^{3} \mathrm{~J}(\mathrm{H}, \mathrm{H})=8.5\right.$, $\left.{ }^{4} \mathrm{~J}(\mathrm{H}, \mathrm{H})=3.3,6 \mathrm{H}, \mathrm{C}_{6} \mathrm{H}_{4}-\mathrm{CH}_{2}\right), 0.83\left(\mathrm{dt},{ }^{3} \mathrm{~J}(\mathrm{H}, \mathrm{H})=8.5,{ }^{4} \mathrm{~J}(\mathrm{H}, \mathrm{H})=4.1,6 \mathrm{H}, \mathrm{Si}-\mathrm{CH}_{2}\right)$, 0.35 (br s, $12 \mathrm{H}, \mathrm{Si}-\mathrm{CH}_{2}$ ), -0.02 (br s, $\left.18 \mathrm{H}, \mathrm{Si}-\mathrm{CH}_{3}\right) .{ }^{1} \mathrm{H}\left\{{ }^{11} \mathrm{~B}\right\} \mathrm{NMR}: \delta$ 7.69-7.34 (m, 5H, $\left.\mathrm{C}_{6} H_{5}\right), 7.33\left(\mathrm{~d},{ }^{3} \mathrm{~J}(\mathrm{H}, \mathrm{H})=8.5,6 \mathrm{H}, \mathrm{C}_{6} \mathrm{H}_{4}\right), 7.17\left(\mathrm{~d},{ }^{3} \mathrm{~J}(\mathrm{H}, \mathrm{H})=4.1,6 \mathrm{H}, \mathrm{C}_{6} H_{4}\right), 4.59(\mathrm{~s}$, $\left.6 \mathrm{H}, \mathrm{CH}_{2}-\mathrm{Cl}\right), 2.58\left(\mathrm{tt},{ }^{3} \mathrm{~J}(\mathrm{H}, \mathrm{H})=8.5,{ }^{4} \mathrm{~J}(\mathrm{H}, \mathrm{H})=3.3,6 \mathrm{H}, \mathrm{C}_{6} \mathrm{H}_{4}-\mathrm{CH}_{2}\right), 2.91$ (br s, B- $H$ ), 2.38 (br s, B-H), 2.28 (br s, B-H), $00.83\left(\mathrm{dt},{ }^{3} \mathrm{~J}(\mathrm{H}, \mathrm{H})=8.5,{ }^{4} \mathrm{~J}(\mathrm{H}, \mathrm{H})=4.1,6 \mathrm{H}, \mathrm{Si}-\mathrm{CH}_{2}\right)$, 0.35 (br s, $12 \mathrm{H}, \mathrm{Si}-\mathrm{CH}_{2}$ ), -0.02 (br s, $\left.18 \mathrm{H}, \mathrm{Si}-\mathrm{CH}_{3}\right) .{ }^{11} \mathrm{~B}$ NMR: $\delta 3.45$ (br, 1B), -1.2 (br,1B), -6.2 (br, 8B). ${ }^{13} \mathrm{C}\left\{{ }^{1} \mathrm{H}\right\}$ NMR: $\delta$ 145.3, 128.7, 128.5, $128.1\left(C_{6} \mathrm{H}_{4}\right), 133.1,131.3$, 130.6, $128.2\left(C_{6} \mathrm{H}_{5}\right), 83.8$ ( $\left.C_{\text {cluster }}\right), 76.1\left(C_{\text {cluster }}\right), 46.2\left(\mathrm{CH}_{2}-\mathrm{Cl}\right), 29.7\left(\mathrm{C}_{6} \mathrm{H}_{4}-\mathrm{CH}_{2}\right), 16.5$ $\left(\mathrm{Si}-\mathrm{CH}_{2}\right), 7.4\left(\mathrm{Si}-\mathrm{CH}_{2}\right), 4.6\left(\mathrm{Si}-\mathrm{CH}_{2}\right),-4.0\left(\mathrm{Si}-\mathrm{CH}_{3}\right) .{ }^{29} \mathrm{Si}\left\{{ }^{1} \mathrm{H}\right\} \mathrm{NMR}: \delta 10.38\left(\mathrm{Si}-\mathrm{C}_{\text {cluster }}\right)$, 
$4.72\left(\mathrm{Si}_{-}-\mathrm{CH}_{2}\right)$. FTIR $(\mathrm{NaCl}), \mathrm{cm}^{-1}: 3053\left(v\left(\mathrm{C}_{\text {aryl }}-\mathrm{H}\right)\right), 2952-2923\left(v\left(\mathrm{C}_{\text {alkyl }}-\mathrm{H}\right)\right), 2586$ $(v(\mathrm{~B}-\mathrm{H})), 1247\left(\delta\left(\mathrm{Si}_{-} \mathrm{CH}_{3}\right)\right)$. Anal. Calcd for $\mathrm{C}_{47} \mathrm{H}_{75} \mathrm{~B}_{10} \mathrm{Si}_{4} \mathrm{Cl}_{3}: \mathrm{C}, 58.38 ; \mathrm{H}, 7.88 \%$. Found: $\mathrm{C}, 57.91 ; \mathrm{H}, 7.97$. MS-Electrospray (ESI) solution of $\mathrm{CHCl}_{3} / \mathrm{MeOH} \mathrm{m} / \mathrm{z}$ calcd for $\mathrm{C}_{47} \mathrm{H}_{75} \mathrm{~B}_{10} \mathrm{Si}_{4} \mathrm{Cl}_{3}$ : 966.9; found: $984.4\left[\left(\mathrm{M}+\mathrm{H}_{2} \mathrm{O}\right)^{+}, 49 \%\right]$

\section{Synthesis of 1- $\mathrm{CH}_{3}-2-\left[\mathrm{Si}\left(\mathrm{CH}_{2} \mathrm{CH}_{2}\left(\mathrm{CH}_{3}\right)_{2} \mathrm{Si}\left(1^{\prime}-\mathrm{CH}_{3}-1^{\prime}, 2^{\prime}-\mathrm{C}_{2} \mathrm{~B}_{10} \mathrm{H}_{10}\right)_{3}\right]-1,2-\mathrm{C}_{2} \mathrm{~B}_{10} \mathrm{H}_{10}\right.$} (12). To a solution of $1-\mathrm{CH}_{3}-1,2-\mathrm{C}_{2} \mathrm{~B}_{10} \mathrm{H}_{11}(0.33 \mathrm{~g}, 2.09 \mathrm{mmol})$ in $\mathrm{Et}_{2} \mathrm{O}(3 \mathrm{~mL})$ and toluene $(6 \mathrm{~mL})$ at $0^{\circ} \mathrm{C}$ was added drop-wise a $1.6 \mathrm{M}$ solution of $n$-BuLi in hexane $(1.4$ $\mathrm{mL}, 2.24 \mathrm{mmol})$. The mixture was stirred for $1 \mathrm{~h}$ at room temperature. After cooling it to $0^{\circ} \mathrm{C}$, a solution of $5(0.38 \mathrm{~g}, 0.70 \mathrm{mmol})$ in toluene $(6 \mathrm{~mL})$ and $\mathrm{Et}_{2} \mathrm{O}(3 \mathrm{~mL})$ was added drop-wise with stirring. The suspension was stirred for $2 \mathrm{~h}$ at room temperature and was filtered off through Celite. The solvent was removed and the residue was treated with $5 \mathrm{~mL}$ of cold $\mathrm{Et}_{2} \mathrm{O}$ to isolate 12 as a white solid. Yield: $0.13 \mathrm{~g}, 20.3 \% .{ }^{1} \mathrm{H}$ NMR: $\delta 2.02\left(\mathrm{~s}, 12 \mathrm{H}, \mathrm{C}_{\text {cluster }}-\mathrm{CH}_{3}\right), 0.82\left(\mathrm{~m}, 12 \mathrm{H}, \mathrm{Si}-\mathrm{CH}_{2}\right), 0.38\left(\mathrm{~s}, 18 \mathrm{H}, \mathrm{Si}-\mathrm{CH}_{3}\right)$. ${ }^{1} \mathrm{H}\left\{{ }^{11} \mathrm{~B}\right\}$ NMR: $\delta 2.46$ (br s, B-H), 2.27 (br s, B-H), 2.02 (s, 12H, $\left.\mathrm{C}_{\text {cluster }}-\mathrm{CH}_{3}\right), 0.82$ (m, 12H, Si- $\mathrm{CH}_{2}$ ), $0.38\left(\mathrm{~s}, 18 \mathrm{H}, \mathrm{Si}-\mathrm{CH}_{3}\right) .{ }^{11} \mathrm{~B}$ NMR: $\delta 2.4$ (br, 1B), -3.3 (br, 1B), -6.5 (br, 4B), -7.5 (br, 2B), -11.2 (br, 2B). ${ }^{13} \mathrm{C}\left\{{ }^{1} \mathrm{H}\right\}$ NMR: $\delta 75.3$ ( $\left.C_{\text {cluster }}\right), 70.3\left(C_{\text {cluster }}\right), 25.8$ $\left(\mathrm{C}_{\text {cluster- }}-\mathrm{CH}_{3}\right), 9.5\left(\mathrm{Si}-\mathrm{CH}_{2}\right), \quad 5.5\left(\mathrm{Si}-\mathrm{CH}_{2}\right),-2.2\left(\mathrm{Si}-\mathrm{CH}_{3}\right) .{ }^{29} \mathrm{Si}\left\{{ }^{1} \mathrm{H}\right\} \quad \mathrm{NMR}: \delta \quad 9.00$ $\left(S i_{\text {periphery }}\right), 8.06\left(\mathrm{Si}_{\text {core }}\right)$. FTIR $(\mathrm{KBr}), \mathrm{cm}^{-1}: 2951\left(v\left(\mathrm{C}_{\text {alkyl }}-\mathrm{H}\right)\right), 2582(v(\mathrm{~B}-\mathrm{H})), 1258$ $\left(\delta\left(\mathrm{Si}-\mathrm{CH}_{3}\right)\right)$. Anal. Calcd. for $\mathrm{C}_{24} \mathrm{H}_{82} \mathrm{~B}_{40} \mathrm{Si}_{4}: \mathrm{C}, 31.48 ; \mathrm{H}, 9.03$. Found: C, 32.06; H, 9.03. MS-Electrospray (ESI) solution of $\mathrm{CHCl}_{3} / \mathrm{MeOH} \mathrm{m} / \mathrm{z}$ calcd for $\mathrm{C}_{24} \mathrm{H}_{82} \mathrm{~B}_{40} \mathrm{Si}_{4}$ : 915.7 ; found, $938.8\left[(\mathrm{M}+\mathrm{Na})^{+}, 39 \%\right]$.

\section{Synthesis of 1- $\mathrm{C}_{6} \mathrm{H}_{5}-2-\left[\mathrm{Si}\left(\mathrm{CH}_{2} \mathrm{CH}_{2} \mathrm{Si}\left(\mathrm{CH}_{3}\right)_{2}\left(1^{\prime}-\mathrm{C}_{6} \mathrm{H}_{5}-1^{\prime}, 2^{\prime}-\mathrm{C}_{2} \mathrm{~B}_{10} \mathrm{H}_{10}\right)_{3}\right]-1,2-\mathrm{C}_{2} \mathrm{~B}_{10} \mathrm{H}_{10}\right.$}

(13). The procedure was the same as for 12 using $1-\mathrm{C}_{6} \mathrm{H}_{5}-1,2-\mathrm{C}_{2} \mathrm{~B}_{10} \mathrm{H}_{11}(0.19 \mathrm{~g}, 0.87$ 
mmol) and $n$-BuLi $(0.59 \mathrm{~mL}, 0.94 \mathrm{mmol})$ in toluene $(2 \mathrm{~mL})$ and $\mathrm{Et}_{2} \mathrm{O}(1 \mathrm{~mL})$. After cooling at $0^{\circ} \mathrm{C}$, a solution of $6(0.18 \mathrm{~g}, 0.29 \mathrm{mmol})$ in toluene $(2 \mathrm{~mL})$ and $\mathrm{Et}_{2} \mathrm{O}(1 \mathrm{~mL})$ was added drop-wise with stirring. The suspension was stirred for $5 \mathrm{~h}$ at room temperature and filtered off through Celite. The solvent was removed to vacuum to give a brown oil, which was treated with hexane to obtain $\mathbf{1 3}$ as a brown oil. Yield: $0.08 \mathrm{~g}$, 24.5\%. ${ }^{1} \mathrm{H}$ NMR: $\delta$ 7.68-7.36 (m, 20H, $\left.\mathrm{C}_{6} H_{5}\right), 0.21\left(\mathrm{~m}, 12 \mathrm{H}, \mathrm{Si}-\mathrm{CH}_{2}\right),-0.19(\mathrm{~s}, 18 \mathrm{H}, \mathrm{Si}-$ $\left.\mathrm{CH}_{3}\right) .{ }^{1} \mathrm{H}\left\{{ }^{11} \mathrm{~B}\right\}$ NMR: $\delta$ 7.68-7.36 (m, 20H, $\left.\mathrm{C}_{6} H_{5}\right), 2.89$ (br s, B-H), 2.62 (br s, B-H), 2.35 (br s, B- $H$ ), 0.21 (m, 12H, Si-CH2), -0.19 (s, 18H, Si-CH $\left.H_{3}\right) .{ }^{11}$ B NMR: $\delta 3.3$ (br, 1B), -1.0 (br, 1B), -6.4 (br, 4B), -9.3 (br, 4B). ${ }^{13} \mathrm{C}\left\{{ }^{1} \mathrm{H}\right\}$ NMR: $\delta$ 132.6-128.5 $\left(C_{6} \mathrm{H}_{5}\right)$, $83.5\left(C_{\text {cluster }}\right), 75.4\left(C_{\text {cluster }}\right), 8.5\left(\mathrm{Si}-C_{2}\right), 4.3\left(\mathrm{Si}-\mathrm{CH}_{2}\right),-3.4\left(\mathrm{Si}-\mathrm{CH}_{3}\right) .{ }^{29} \mathrm{Si}\left\{{ }^{1} \mathrm{H}\right\} \mathrm{NMR}: \delta$ $10.32\left(S_{i_{\text {periphery }}}\right), 10.02\left(\mathrm{Si}_{\text {core }}\right)$. FTIR $(\mathrm{KBr}), \mathrm{cm}^{-1}: 3074\left(v\left(\mathrm{C}_{\text {aryl }}-\mathrm{H}\right)\right), 2960-2926$ $\left(v\left(\mathrm{C}_{\text {alkyl }}-\mathrm{H}\right)\right), 2583(v(\mathrm{~B}-\mathrm{H})), 1259\left(\delta\left(\mathrm{Si}_{-} \mathrm{CH}_{3}\right)\right)$. Anal. Calcd. for $\mathrm{C}_{44} \mathrm{H}_{90} \mathrm{~B}_{40} \mathrm{Si}_{4}: \mathrm{C}, 45.40$; $\mathrm{H}, 7.79$. Found: $\mathrm{C}, 44.92 ; \mathrm{H}, 8.19$. MS-Electrospray (ESI) solution of $\mathrm{CHCl}_{3} / \mathrm{MeOH}$ $\mathrm{m} / \mathrm{z}$ calcd for $\mathrm{C}_{44} \mathrm{H}_{90} \mathrm{~B}_{40} \mathrm{Si}_{4}:$ 1164.0; found, 1187.0 [(M $\left.\left.+\mathrm{Na}\right)^{+}, 37 \%\right]$.

\section{Synthesis of $\left[\mathrm{N}(\mathrm{Me})_{4}\right]\left[7-\mathrm{Si}\left(\mathrm{CH}=\mathrm{CH}_{2}\right)_{3}-\mu-\left(9,10-\mathrm{CHSi}\left(\mathrm{CH}=\mathrm{CH}_{2}\right)_{3}\right)-\right.$ nido- $\mathrm{CB}_{10} \mathrm{H}_{10}(14)$.}

In a Schlenk flask were added Mg (1.25 g, $0.05 \mathrm{mmol}), 1 \mathrm{~mL}$ of THF and a crystal of $\mathrm{I}_{2}$. Then a solution of $1(0.25 \mathrm{~g}, 0.69 \mathrm{mmol})$ in THF $(5 \mathrm{~mL})$ and dibromoethane $(1.3 \mathrm{~mL}$, $3.17 \mathrm{mmol}$ ) were added drop-wise at the same time to the flask. After the addition was completed, the reaction mixture was refluxed for $15 \mathrm{~h}$ and cooled at room temperature. The volatile compounds were removed in vacuo and the reaction residue washed with water $(50 \mathrm{~mL})$ and filtered. An excess of $\left[\mathrm{N}\left(\mathrm{CH}_{3}\right)_{4}\right] \mathrm{Cl}$ in water was added to precipitate a white solid. The solid was filtered off, washed with water and dried under vacuum to obtain 14. Yield: $93 \mathrm{mg}, 31 \% .{ }^{1} \mathrm{H}$ NMR: $\delta$ 6.34-5.79 (m, $\left.18 \mathrm{H}, \mathrm{CH}=\mathrm{CH}_{2}\right), 3.80(\mathrm{~s}, 1 \mathrm{H}$, $\mathrm{C}-H), 3.45\left(\mathrm{~s}, 12 \mathrm{H},\left(\left[\mathrm{N}\left(\mathrm{CH}_{3}\right)_{4}\right]\right) .{ }^{1} \mathrm{H}\left\{{ }^{11} \mathrm{~B}\right\} \mathrm{NMR}: \delta 6.34-5.787\left(\mathrm{~m}, 18 \mathrm{H}, \mathrm{C} H=\mathrm{CH}_{2}\right), 4.22\right.$ 
(br s, 2H, B-H), 3.90 (s, 1H, Si-C-H), 3.78 (br s, 1H, B- $H), 3.45$ (s, 12H, ([N(CH $\left.\left.\left(\mathrm{CH}_{3}\right)_{4}\right]\right)$, 3.05 (br s, 2H, B- $H$ ), 2.20 (br s, 2H, B-H), 1.67 (br s, 2H, B-H), 0.99 (br s, 2H, B-H). ${ }^{11}$ B NMR: $\delta 20.3\left(\mathrm{~d},{ }^{1} \mathrm{~J}(\mathrm{~B}, \mathrm{H})=139,2 \mathrm{~B}\right), 17.4\left(\mathrm{~d},{ }^{1} \mathrm{~J}(\mathrm{~B}, \mathrm{H})=143,1 \mathrm{~B}\right), 4.7\left(\mathrm{~d},{ }^{1} \mathrm{~J}(\mathrm{~B}, \mathrm{H})=\right.$ $142,1 \mathrm{~B}),-4.9\left(\mathrm{~d},{ }^{1} \mathrm{~J}(\mathrm{~B}, \mathrm{H})=149,2 \mathrm{~B}\right),-12.6\left(\mathrm{~d},{ }^{1} \mathrm{~J}(\mathrm{~B}, \mathrm{H})=136,2 \mathrm{~B}\right),-20 .\left(\mathrm{d},{ }^{1} \mathrm{~J}(\mathrm{~B}, \mathrm{H})=\right.$ 134, 2B). ${ }^{13} \mathrm{C}\left\{{ }^{1} \mathrm{H}\right\}$ NMR: $\delta$ 138.4-131.7 $\left(\mathrm{CH}=\mathrm{CH}_{2}\right), 81.7(\mathrm{CHSi}), 55.2\left(\left[\mathrm{~N}\left(\mathrm{CH}_{3}\right)_{4}\right]\right)$. FTIR (KBr), cm ${ }^{-1}: 3051(v(\mathrm{C}-\mathrm{H})), 2945\left(v\left(\mathrm{C}_{\text {alkyl }}-\mathrm{H}\right)\right), 2515(v(\mathrm{~B}-\mathrm{H})), 1404(v(\mathrm{~N}-\mathrm{H}))$. MALDI-TOF-MS (m/z): calcd. 361.66, found 360.36 [M-1, $100 \%$ ], 349.28 [M-BH, 20 \%].

Synthesis of $\left[\mathrm{N}(\mathrm{Me})_{4}\right]\left[7-\mathrm{Si}\left(\mathrm{CH}=\mathrm{CH}_{2}\right)_{3}-\mu-\left(9,10-\mathrm{CHCH}_{3}\right)-\right.$ nido- $\left.\mathrm{CB}_{10} \mathrm{H}_{10}\right]$ (15). The procedure was the same as for $\mathbf{1 4}$ using $\mathrm{Mg}(1.25 \mathrm{~g}, 0.05 \mathrm{mmol})$, a crystal of $\mathrm{I}_{2}$ in THF $(1 \mathrm{~mL})$, a solution of $2(0.23 \mathrm{~g}, 0.86 \mathrm{mmol})$ in THF $(5 \mathrm{~mL})$ and dibromoethane $(1.3$ $\mathrm{mL}, 3.17 \mathrm{mmol}$ ). After $15 \mathrm{~h}$ at reflux the compound $\mathbf{1 5}$ was obtained as a white solid by using the same work up as for 14. Yield: $40.0 \mathrm{mg}, 32 \%$. ${ }^{1} \mathrm{H}$ NMR: $\delta 6.28\left(\mathrm{dd},{ }^{3} \mathrm{~J}(\mathrm{H}, \mathrm{H})=\right.$ $\left.20.2,{ }^{3} \mathrm{~J}(\mathrm{H}, \mathrm{H})=14.6,3 \mathrm{H}, \mathrm{CH}=\mathrm{CH}_{2}\right), 6.03\left(\mathrm{dd},{ }^{3} \mathrm{~J}(\mathrm{H}, \mathrm{H})=14.6,{ }^{2} \mathrm{~J}(\mathrm{H}, \mathrm{H})=4.4,3 \mathrm{H}\right.$, $\left.\mathrm{CH}=\mathrm{CH}_{2}\right), 5.71\left(\mathrm{dd},{ }^{3} \mathrm{~J}(\mathrm{H}, \mathrm{H})=20.2,{ }^{2} \mathrm{~J}(\mathrm{H}, \mathrm{H})=4.4,3 \mathrm{H}, \mathrm{CH}=\mathrm{CH}_{2}\right), 3.68\left(\mathrm{~m}, 1 \mathrm{H}, \mathrm{CH}_{3^{-}}\right.$ $\mathrm{CH}), 3.45\left(\mathrm{~s}, 12 \mathrm{H},\left[\mathrm{N}\left(\mathrm{CH}_{3}\right)_{4}\right]\right), 1.30\left(\mathrm{~s}, 3 \mathrm{H}, \mathrm{CH} \mathrm{H}_{3} \mathrm{CH}\right) .{ }^{1} \mathrm{H}\left\{{ }^{11} \mathrm{~B}\right\}$ NMR: $\delta 6.28$ (dd, $\left.{ }^{3} \mathrm{~J}(\mathrm{H}, \mathrm{H})=20.2,{ }^{3} \mathrm{~J}(\mathrm{H}, \mathrm{H})=14.6,3 \mathrm{H}, \mathrm{CH}=\mathrm{CH}_{2}\right), 6.03\left(\mathrm{dd},{ }^{3} \mathrm{~J}(\mathrm{H}, \mathrm{H})=14.6,{ }^{2} \mathrm{~J}(\mathrm{H}, \mathrm{H})=4.4\right.$, $\left.3 \mathrm{H}, \mathrm{CH}=\mathrm{CH}_{2}\right), 5.71\left(\mathrm{dd},{ }^{3} \mathrm{~J}(\mathrm{H}, \mathrm{H})=20.2,{ }^{2} \mathrm{~J}(\mathrm{H}, \mathrm{H})=4.4,3 \mathrm{H}, \mathrm{CH}=\mathrm{CH}_{2}\right), 3.83($ br s, $2 \mathrm{H}$, B- $H$ ), $3.68\left(\mathrm{~m}, 1 \mathrm{H}, \mathrm{CH}_{3}-\mathrm{CH}\right), 3.64$ (br S, 1H, B- $\left.H\right), 3.45$ (s, 12H, [N(CH $\left.\left.)_{4}\right]\right), 2.70$ (br s, 2H, B-H), 1.87 (br s, 2H, B-H), 1.69 (br s, 2H, B- $H$ ), $1.30\left(\mathrm{~d},{ }^{3} \mathrm{~J}(\mathrm{H}, \mathrm{H})=6.2,3 \mathrm{H}, \mathrm{CH}_{3^{-}}\right.$ $\mathrm{CH}), 1.23$ (br s, 2H, B-H). ${ }^{11} \mathrm{~B}$ NMR: $\delta 18.0\left(\mathrm{~d},{ }^{1} \mathrm{~J}(\mathrm{~B}, \mathrm{H})=143,2 \mathrm{~B}\right), 13.5\left(\mathrm{~d},{ }^{1} \mathrm{~J}(\mathrm{~B}, \mathrm{H})=\right.$ $135,1 \mathrm{~B}), 1.1\left(\mathrm{~d},{ }^{1} \mathrm{~J}(\mathrm{~B}, \mathrm{H})=130,1 \mathrm{~B}\right),-5.7\left(\mathrm{~d},{ }^{1} \mathrm{~J}(\mathrm{~B}, \mathrm{H})=145,2 \mathrm{~B}\right),-10.3\left(\mathrm{~d},{ }^{1} \mathrm{~J}(\mathrm{~B}, \mathrm{H})=\right.$ 132, 2B), -16.2. (d, $\left.{ }^{1} \mathrm{~J}(\mathrm{~B}, \mathrm{H})=137,2 \mathrm{~B}\right) .{ }^{13} \mathrm{C} \mathrm{NMR}: \delta 136.4\left(\mathrm{~d},{ }^{1} \mathrm{~J}(\mathrm{C}, \mathrm{H})=136\right.$, $\left.\mathrm{CH}=\mathrm{CH}_{2}\right), 132.6\left(\mathrm{t},{ }^{1} \mathrm{~J}(\mathrm{C}, \mathrm{H})=165, \mathrm{CH}=\mathrm{CH}_{2}\right), 91,1\left(\right.$ br d, $\left.{ }^{1} \mathrm{~J}(\mathrm{C}, \mathrm{H})=168, \mathrm{CH}_{3}-\mathrm{CH}\right)$, 
$55.1\left(\mathrm{q},{ }^{1} \mathrm{~J}(\mathrm{C}, \mathrm{H})=143,\left[\mathrm{~N}\left(\mathrm{CH}_{3}\right)_{4}\right]\right), 32.1\left(\mathrm{q},{ }^{1} \mathrm{~J}(\mathrm{C}, \mathrm{H})=136, \mathrm{CHCH}_{3}\right)$. FTIR $(\mathrm{KBr}), \mathrm{cm}^{-1}$ : $3049(v(\mathrm{C}-\mathrm{H})), 2941\left(v\left(\mathrm{C}_{\text {alkyl }}-\mathrm{H}\right)\right), 2515(v(\mathrm{~B}-\mathrm{H})), 1400(v(\mathrm{~N}-\mathrm{H}))$. MALDI-TOF-MS $(\mathrm{m} / \mathrm{z})$ : calcd. 267.26, found 266.22 [M-1, $100 \%]$.

Synthesis of $\left[\mathrm{N}(\mathrm{Me})_{4}\right]\left[7-\mathrm{Si}\left(\mathrm{CH}=\mathrm{CH}_{2}\right)_{3}-\boldsymbol{\mu}-\left(\mathbf{9}, \mathbf{1 0}-\mathrm{CHC}_{6} \mathrm{H}_{5}\right)-\right.$ nido $-\mathrm{CB}_{10} \mathrm{H}_{10}$ (16). The procedure was the same as for $\mathbf{1 4}$ using $\mathrm{Mg}(1.25 \mathrm{~g}, 0.05 \mathrm{mmol})$, a crystal of $\mathrm{I}_{2}$ in THF $(1 \mathrm{~mL})$, a solution of $3(0.25 \mathrm{~g}, 0.76 \mathrm{mmol})$ in THF $(5 \mathrm{~mL})$ and dibromoethane (1.3 $\mathrm{mL}, 3.17 \mathrm{mmol}$ ). After $15 \mathrm{~h}$ at reflux $\mathbf{1 6}$ was obtained as a white solid by using the same work up as for 14. Yield: $45.0 \mathrm{mg}, 32.0 \% .{ }^{1} \mathrm{H}$ NMR: $\delta 7.06\left(\mathrm{~m}, 5 \mathrm{H}, \mathrm{C}_{6} H_{5}\right), 6.39$ (dd, $\left.{ }^{3} \mathrm{~J}(\mathrm{H}, \mathrm{H})=20.2,{ }^{3} \mathrm{~J}(\mathrm{H}, \mathrm{H})=14.6,3 \mathrm{H}, \mathrm{CH}=\mathrm{CH}_{2}\right), 6.13\left(\mathrm{dd},{ }^{3} \mathrm{~J}(\mathrm{H}, \mathrm{H})=14.6,{ }^{2} \mathrm{~J}(\mathrm{H}, \mathrm{H})=4.4\right.$, $\left.3 \mathrm{H}, \mathrm{CH}=\mathrm{CH}_{2}\right), 5.81\left(\mathrm{dd},{ }^{3} \mathrm{~J}(\mathrm{H}, \mathrm{H})=20.2,{ }^{2} \mathrm{~J}(\mathrm{H}, \mathrm{H})=4.4,3 \mathrm{H}, \mathrm{CH}=\mathrm{CH}_{2}\right), 6.41-5.77(\mathrm{~m}$, 9H, $\left.\mathrm{C} H=\mathrm{CH}_{2}\right), 4.48\left(\mathrm{~s}, 1 \mathrm{H}, \mathrm{C}_{6} \mathrm{H}_{5}-\mathrm{CH}\right), 3.45\left(\mathrm{~s}, 12 \mathrm{H},\left[\mathrm{N}\left(\mathrm{CH}_{3}\right)_{4}\right]\right) .{ }^{1} \mathrm{H}\left\{{ }^{11} \mathrm{~B}\right\} \mathrm{NMR}: \delta 7.06$ $\left(\mathrm{m}, 5 \mathrm{H}, \mathrm{C}_{6} H_{5}\right), 6.39\left(\mathrm{dd},{ }^{3} \mathrm{~J}(\mathrm{H}, \mathrm{H})=20.2,{ }^{3} \mathrm{~J}(\mathrm{H}, \mathrm{H})=14.6,3 \mathrm{H}, \mathrm{CH}=\mathrm{CH}_{2}\right), 6.13(\mathrm{dd}$, $\left.{ }^{3} \mathrm{~J}(\mathrm{H}, \mathrm{H})=14.6,{ }^{2} \mathrm{~J}(\mathrm{H}, \mathrm{H})=4.4,3 \mathrm{H}, \mathrm{CH}=\mathrm{CH}_{2}\right), 5.81\left(\mathrm{dd},{ }^{3} \mathrm{~J}(\mathrm{H}, \mathrm{H})=20.2,{ }^{2} \mathrm{~J}(\mathrm{H}, \mathrm{H})=4.4\right.$, $3 \mathrm{H}, \mathrm{CH}=\mathrm{CH}_{2}$ ), 4.48 (s, $\left.1 \mathrm{H}, \mathrm{C}_{6} \mathrm{H}_{5}-\mathrm{C}-H\right), 3.88$ (br s, B- $H$ ), 3.83 (br s, B- $H$ ), 3.45 (s, $12 \mathrm{H}$, $\left.\left[\mathrm{N}\left(\mathrm{CH}_{3}\right)_{4}\right]\right), 2.77$ (br s, B- $\left.H\right), 1.76$ (br s, B- $\left.H\right), 1.56$ (br s, B-H), 1.29 (br s, B- $\left.H\right) .{ }^{11} \mathrm{~B}$ NMR: $\delta 20.0\left(\mathrm{~d},{ }^{1} \mathrm{~J}(\mathrm{~B}, \mathrm{H})=146,2 \mathrm{~B}\right), 13.8\left(\mathrm{~d},{ }^{1} \mathrm{~J}(\mathrm{~B}, \mathrm{H})=138,1 \mathrm{~B}\right), 2.2\left(\mathrm{~d},{ }^{1} \mathrm{~J}(\mathrm{~B}, \mathrm{H})=\right.$ 133, 1B), -5.6 (d, $\left.{ }^{1} \mathrm{~J}(\mathrm{~B}, \mathrm{H})=139,2 \mathrm{~B}\right),-10.0\left(\mathrm{~d},{ }^{1} \mathrm{~J}(\mathrm{~B}, \mathrm{H})=128,2 \mathrm{~B}\right),-14.1 .\left(\mathrm{d},{ }^{1} \mathrm{~J}(\mathrm{~B}, \mathrm{H})\right.$ $=129,2 \mathrm{~B}) .{ }^{13} \mathrm{C}\left\{{ }^{1} \mathrm{H}\right\}$ NMR: $\delta 136.2\left(\mathrm{CH}=\mathrm{CH}_{2}\right), 133.0\left(\mathrm{CH}=\mathrm{CH}_{2}\right), 128.1-124.6\left(\mathrm{C}_{6} \mathrm{H}_{5}\right)$, $55.1\left(\mathrm{~N}\left(\mathrm{CH}_{3}\right)_{4}\right)$. FTIR $(\mathrm{KBr}), \mathrm{cm}^{-1}: 3051(v(\mathrm{C}-\mathrm{H})), 2958\left(v\left(\mathrm{C}_{\text {alkyl }}-\mathrm{H}\right)\right), 2525(v(\mathrm{~B}-\mathrm{H}))$, $1431(v(\mathrm{~N}-\mathrm{H}))$. MALDI-TOF-MS (m/z): calcd. 329.27, found $327.31(\mathrm{M}-2,100 \%)$; $316.26(\mathrm{M}-\mathrm{B}, 33 \%)$ 


\section{X-ray crystallography:}

Single-crystal data collection for 1-3 were performed at $-100^{\circ} \mathrm{C}$ with an Enraf Nonius KappaCCD diffractometer using graphite monochromatized Mo $\mathrm{K}_{\alpha}$ radiation $(\lambda=$ $0.71073 \AA$ ). A total of 4252,5982 and 7340 unique reflections were collected for $\mathbf{1 - 3}$, respectively. The structures were solved by direct methods and refined on $\mathrm{F}^{2}$ by the SHELXL97 program. ${ }^{1}$ All non-hydrogen atoms were refined with anisotropic displacement parameters, but the hydrogen atoms were treated as riding atoms using the SHELX97 default parameters. Crystallographic parameters for 1-3 are collected in Table 2.

${ }^{1}$ Sheldrick, G. M. SHELX97. University of Göttingen, Germany, 1997. 


\section{${ }^{1} \mathrm{H}\left\{{ }^{11} \mathrm{~B}\right\}$ and ${ }^{13} \mathrm{C}\left\{{ }^{1} \mathrm{H}\right\}$ NMR spectra for compounds 1-16 NMR Spectra of 1}

${ }^{1} \mathrm{H}\left\{{ }^{11} \mathrm{~B}\right\}$-NMR

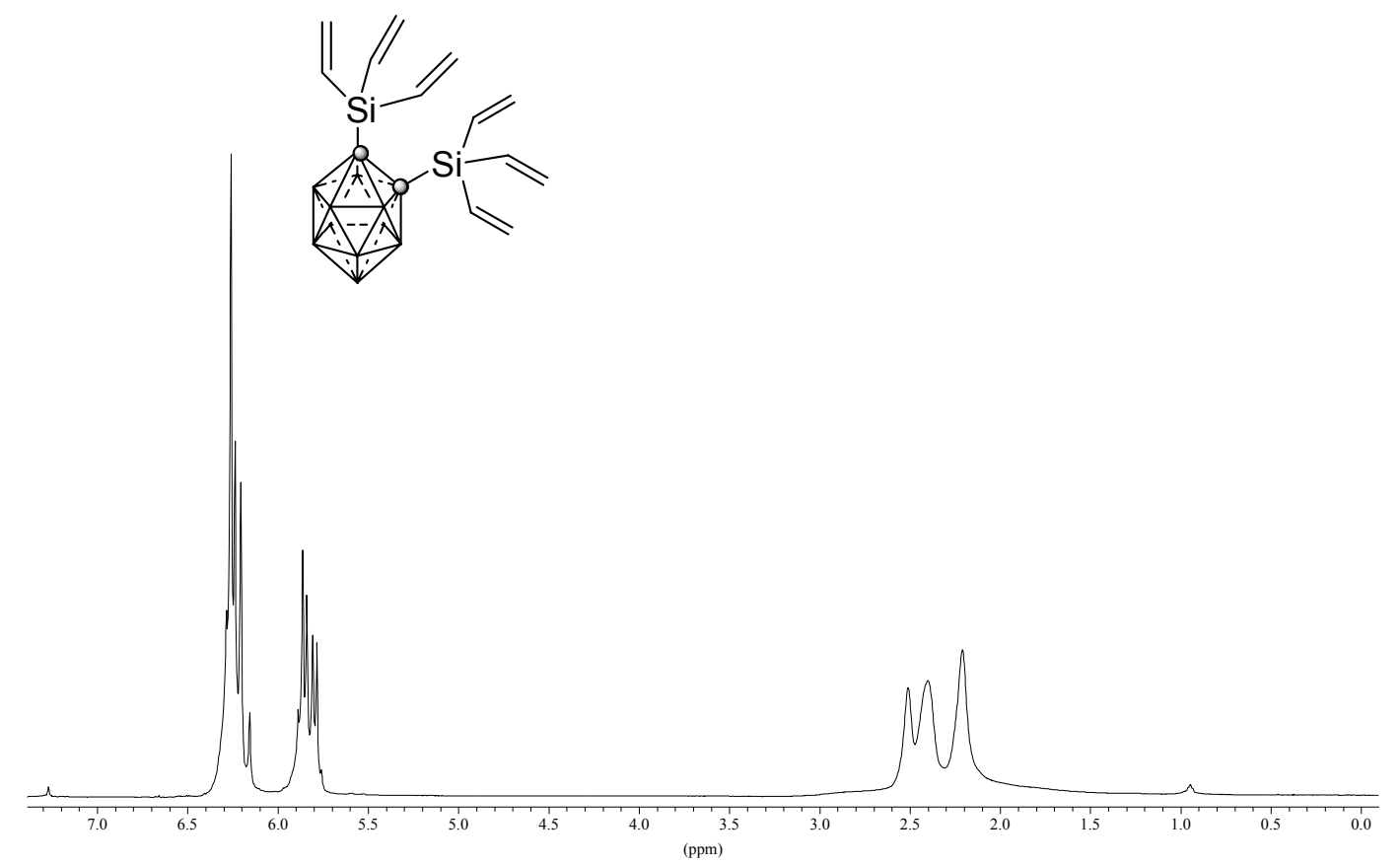

${ }^{13} \mathrm{C}\left\{{ }^{1} \mathrm{H}\right\}-\mathrm{NMR}$

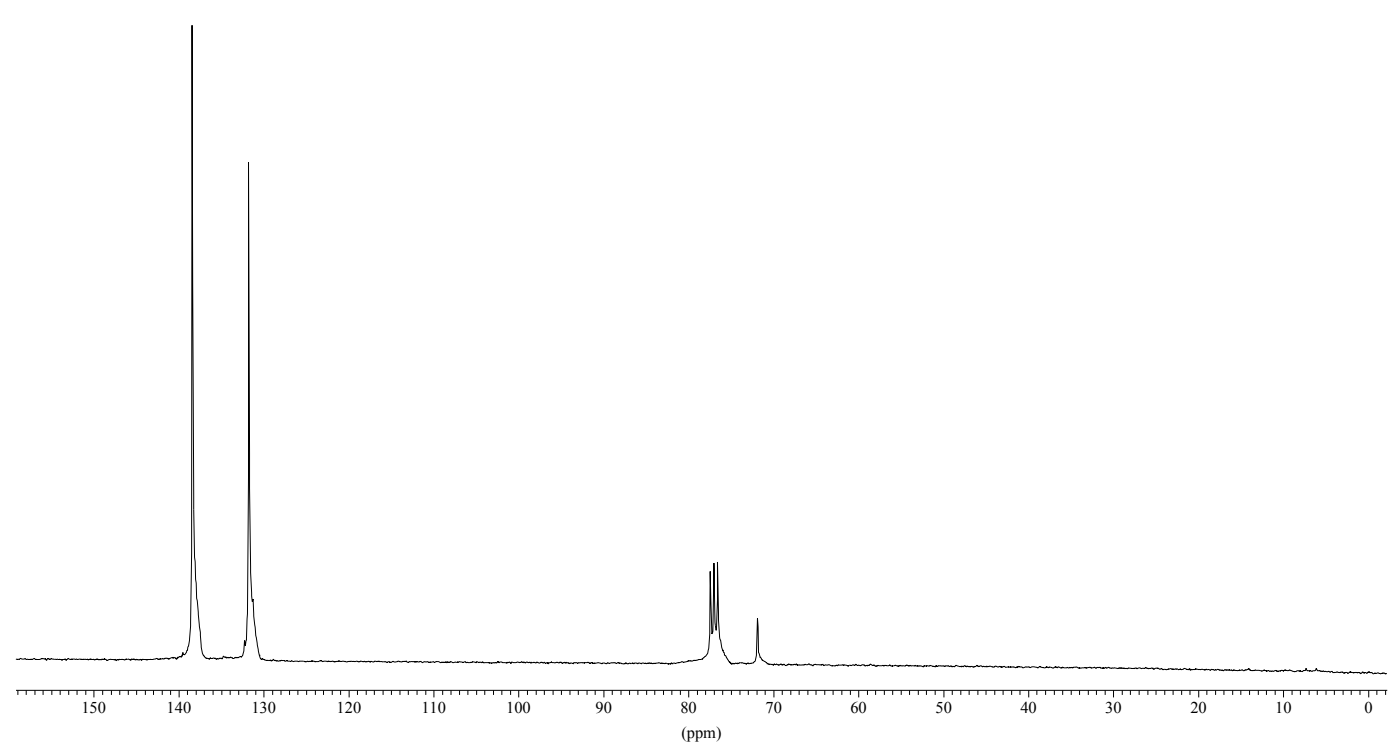


NMR Spectra of 2

\section{${ }^{1} \mathrm{H}\left\{{ }^{11} \mathrm{~B}\right\}-\mathrm{NMR}$}<smiles>C=C[Si](C=C)(C=C)OC(=O)C12C3C4C1C1C2C3C41C</smiles>

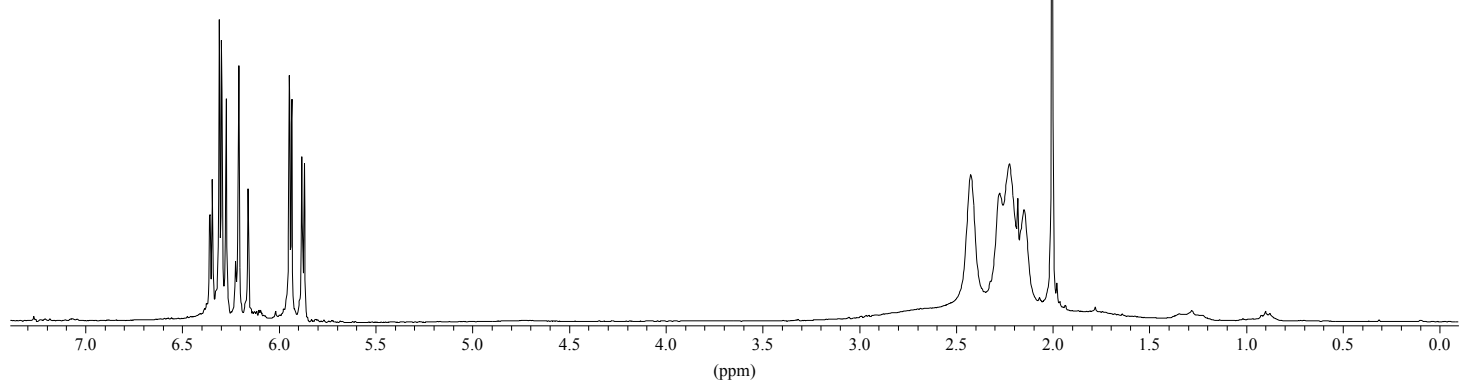

${ }^{13} \mathrm{C}\left\{{ }^{1} \mathrm{H}\right\}-\mathrm{NMR}$

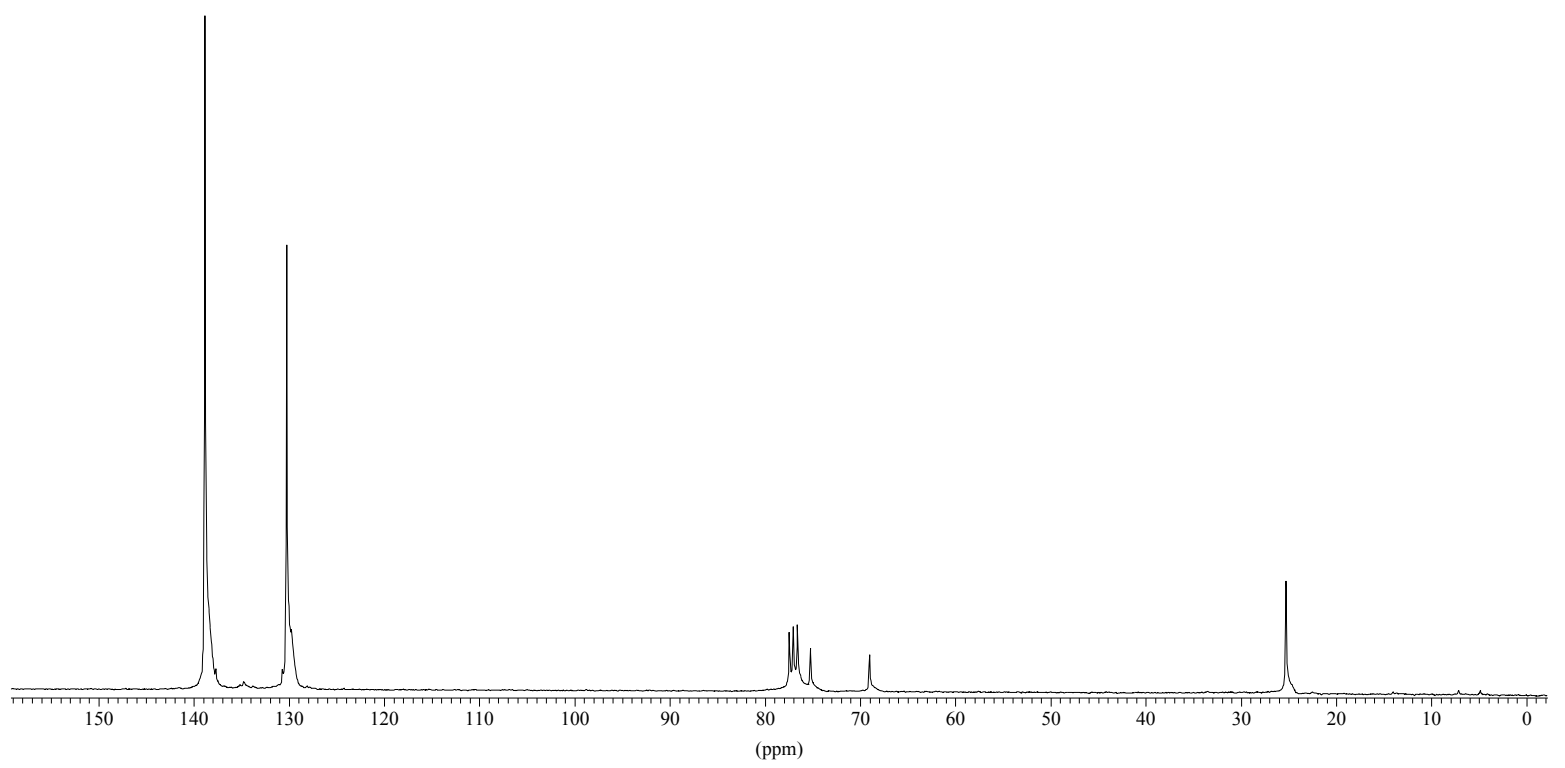


NMR Spectra of 3

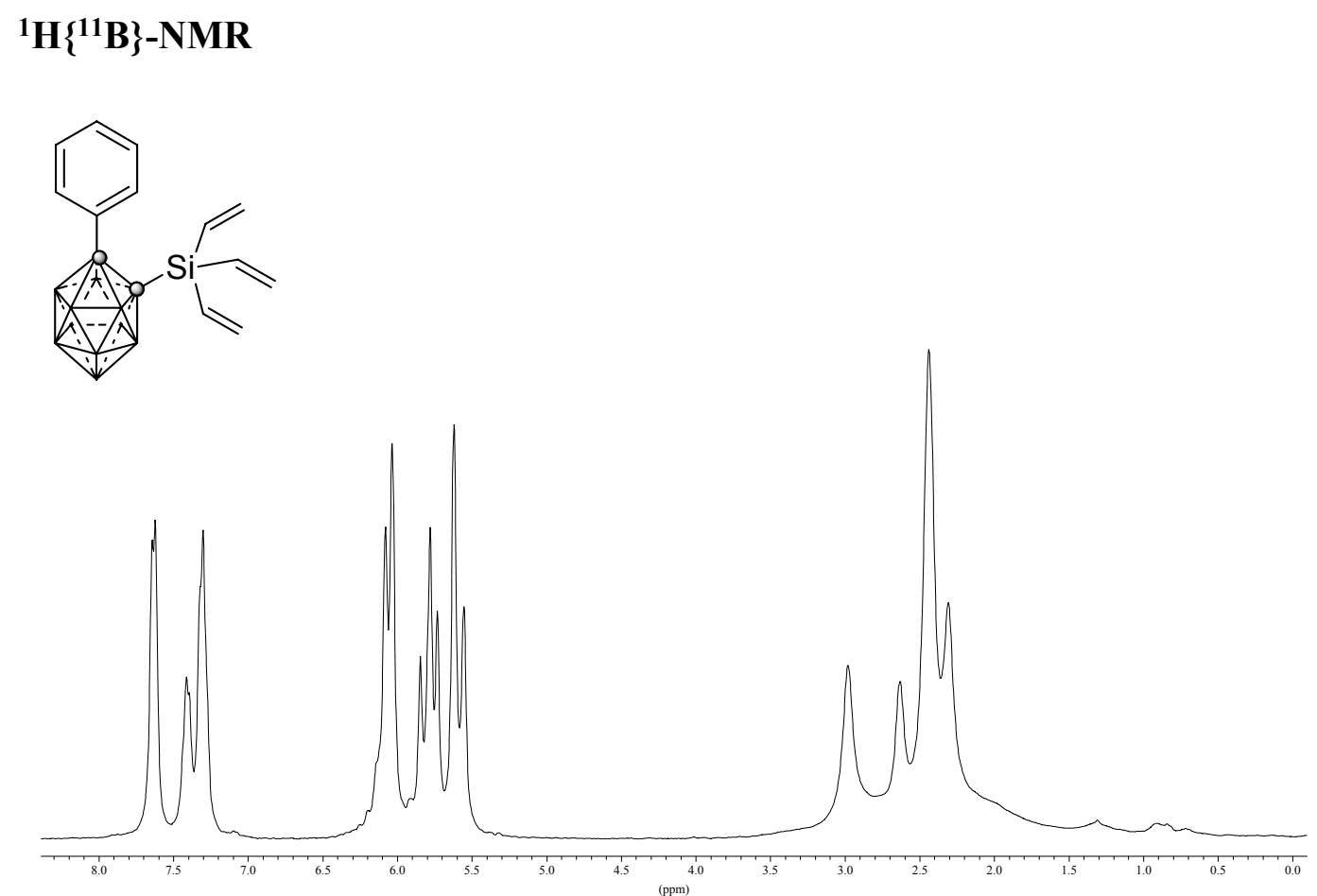

${ }^{13} \mathrm{C}\left\{{ }^{1} \mathrm{H}\right\}-\mathrm{NMR}$

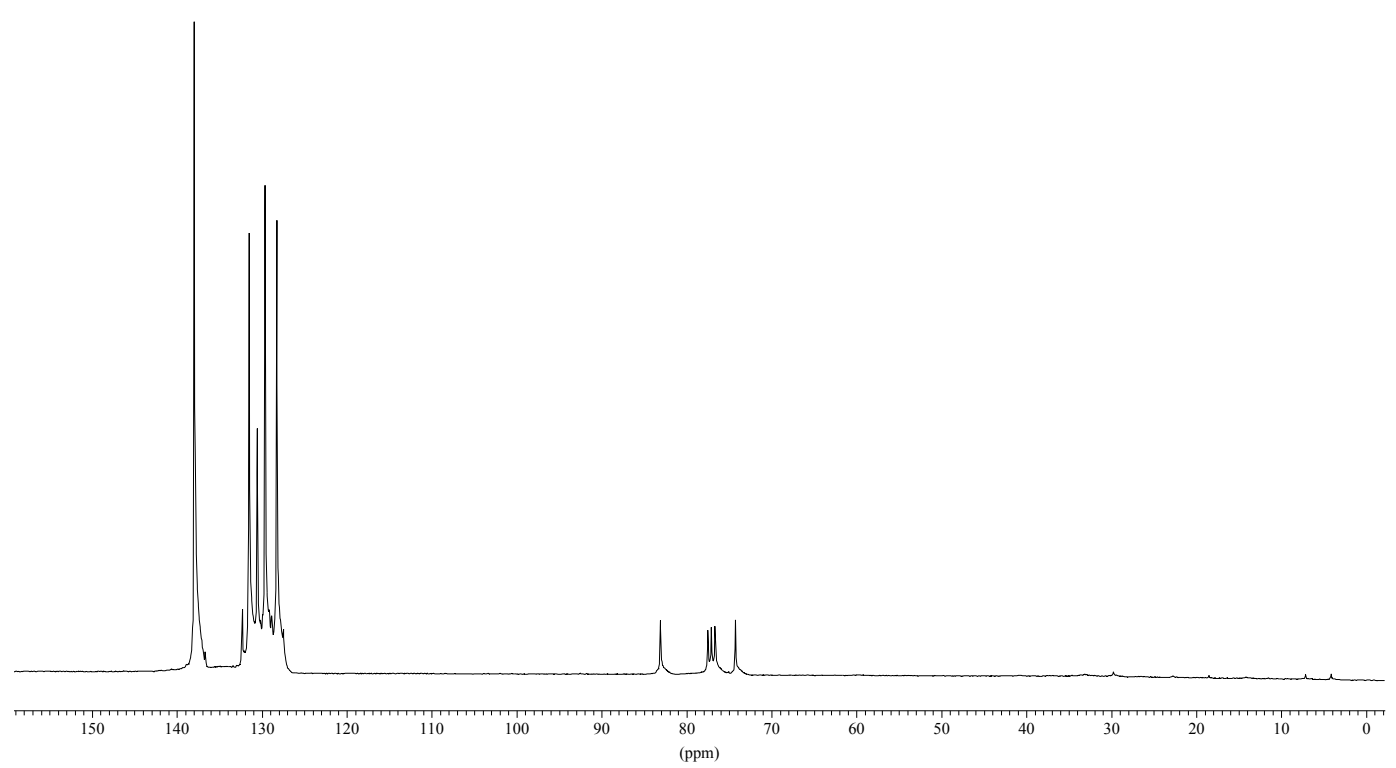




\section{NMR Spectra of 4}

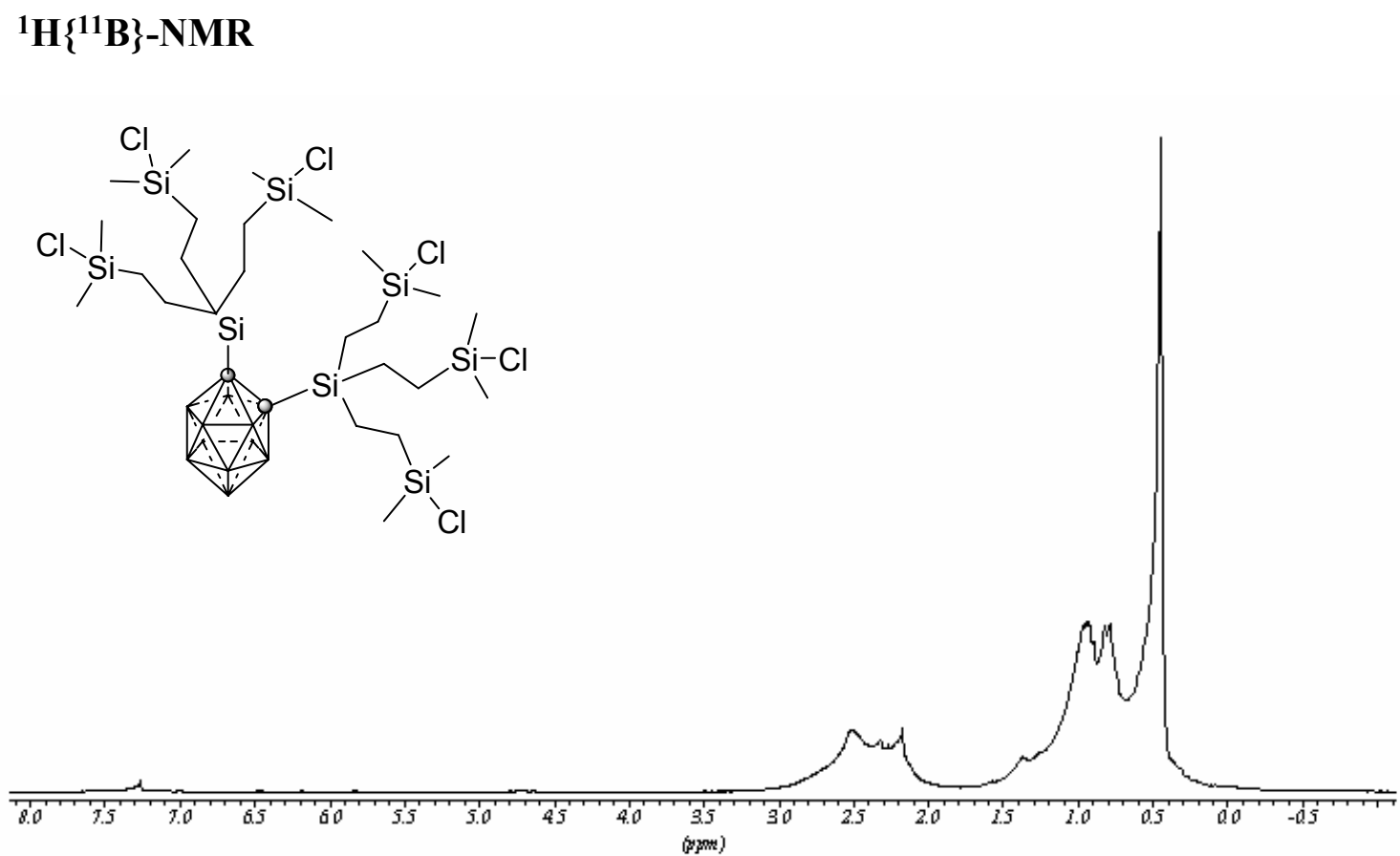

\section{${ }^{13} \mathrm{C}\left\{{ }^{1} \mathrm{H}\right\}-\mathrm{NMR}$}

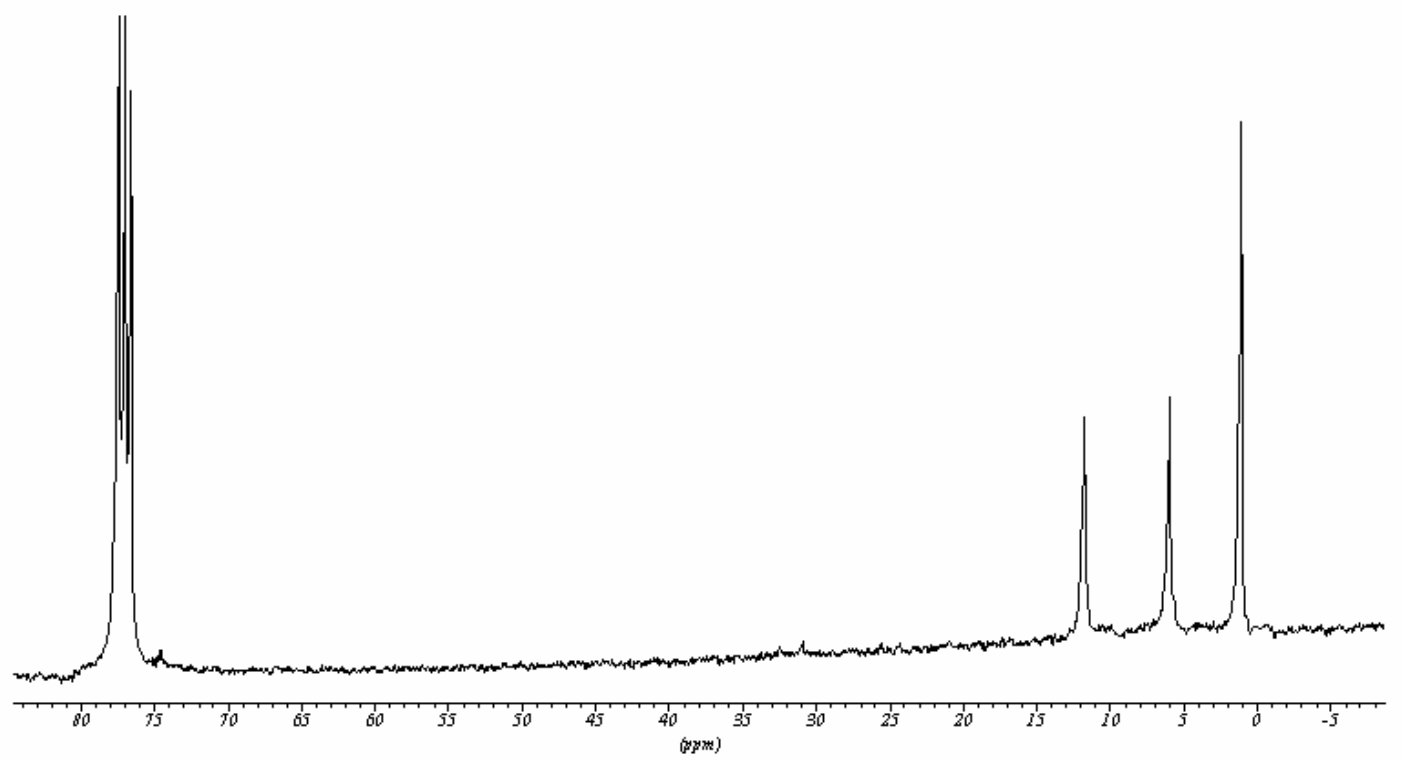




\section{NMR Spectra of 5}

${ }^{1} \mathbf{H}\left\{{ }^{11} B\right\}-N M R$

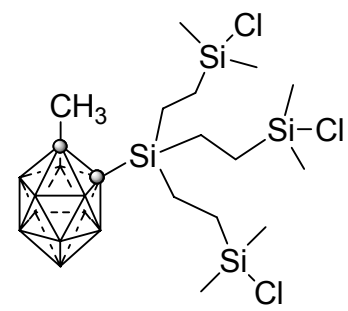

${ }^{13} \mathrm{C}\left\{{ }^{1} \mathrm{H}\right\}-\mathrm{NMR}$ 


\section{NMR Spectra of 6}

\section{${ }^{1} \mathbf{H}\left\{{ }^{11} B\right\}-N M R$}
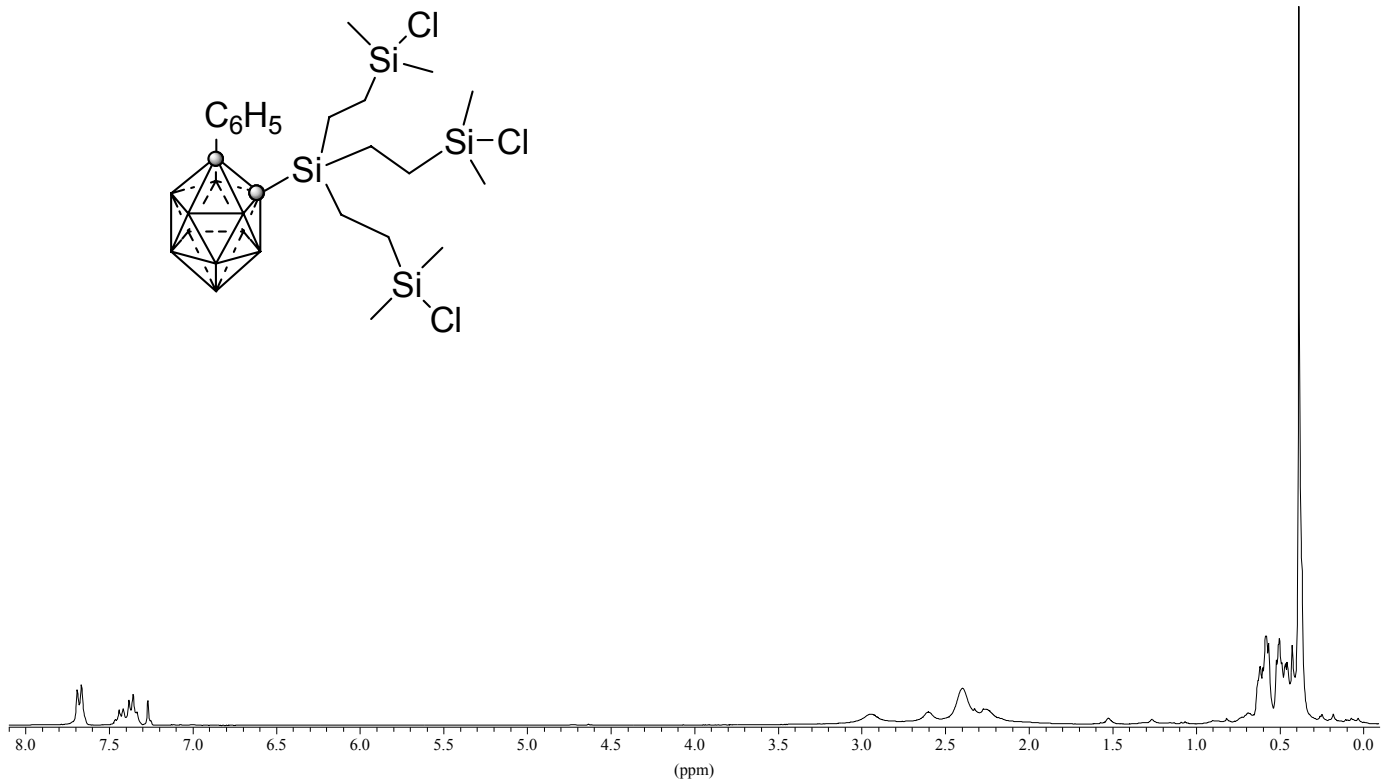

${ }^{13} \mathrm{C}\left\{{ }^{1} \mathrm{H}\right\}-\mathrm{NMR}$

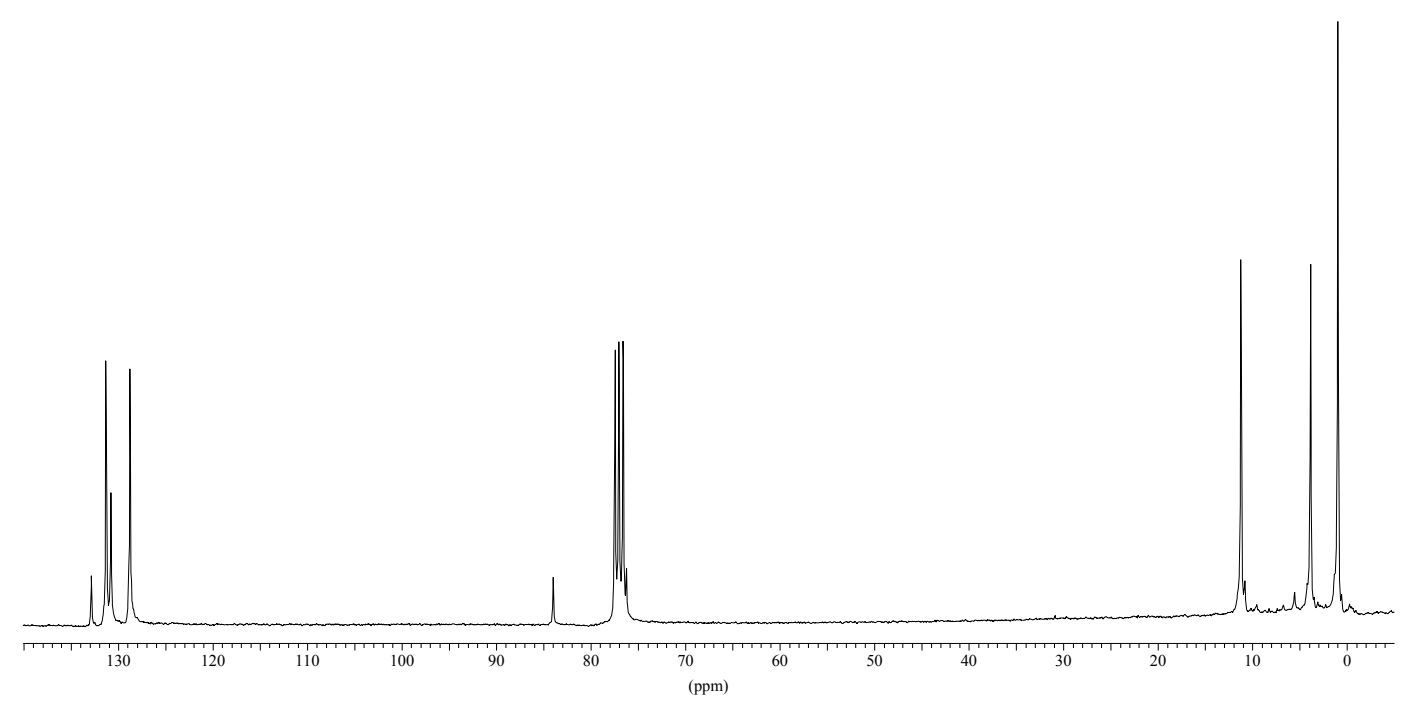




\section{NMR Spectra of 7}

\section{${ }^{1} \mathrm{H}\left\{{ }^{11} \mathrm{~B}\right\}-\mathrm{NMR}$}
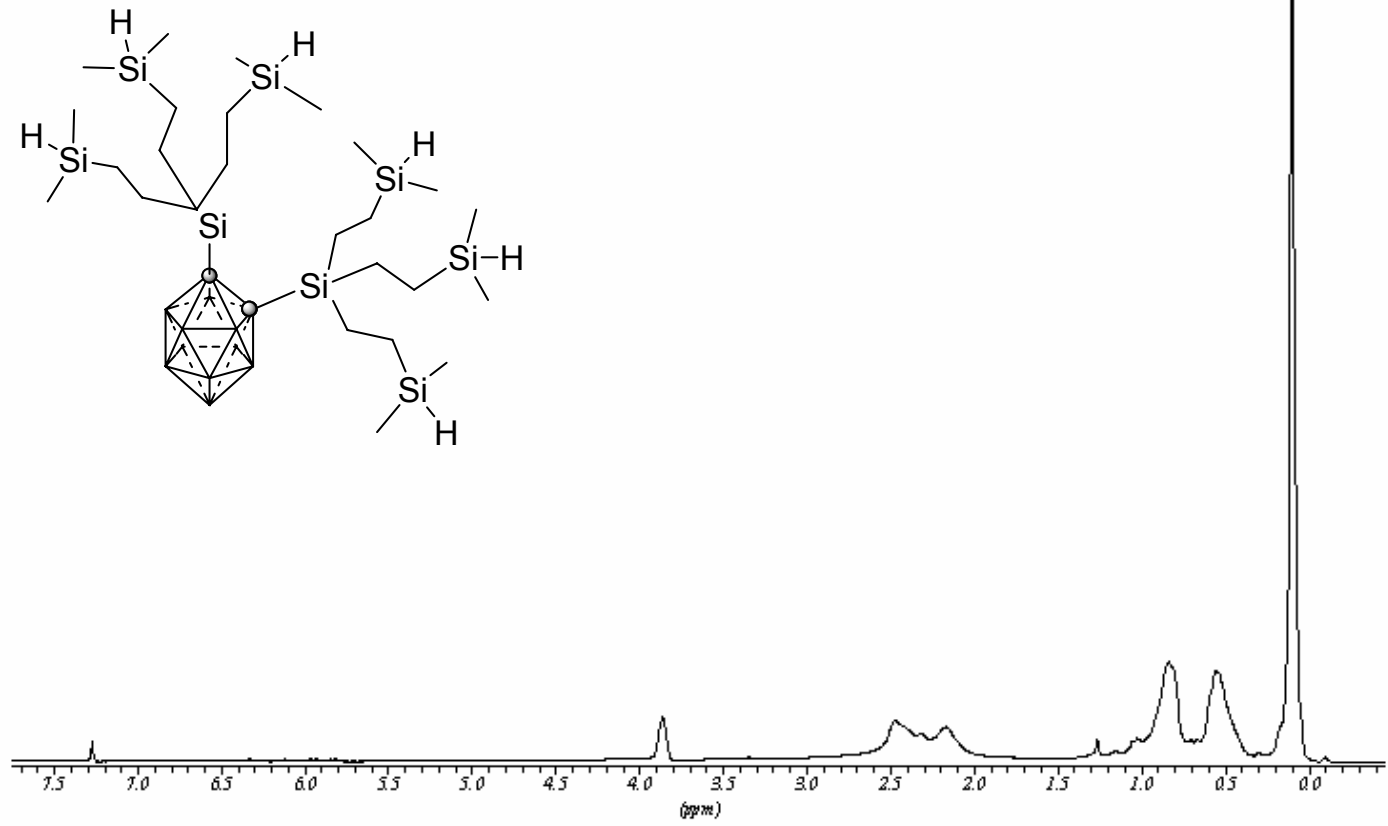

\section{${ }^{13} \mathrm{C}\left\{{ }^{1} \mathrm{H}\right\}-N M R$}


NMR Spectra of 8

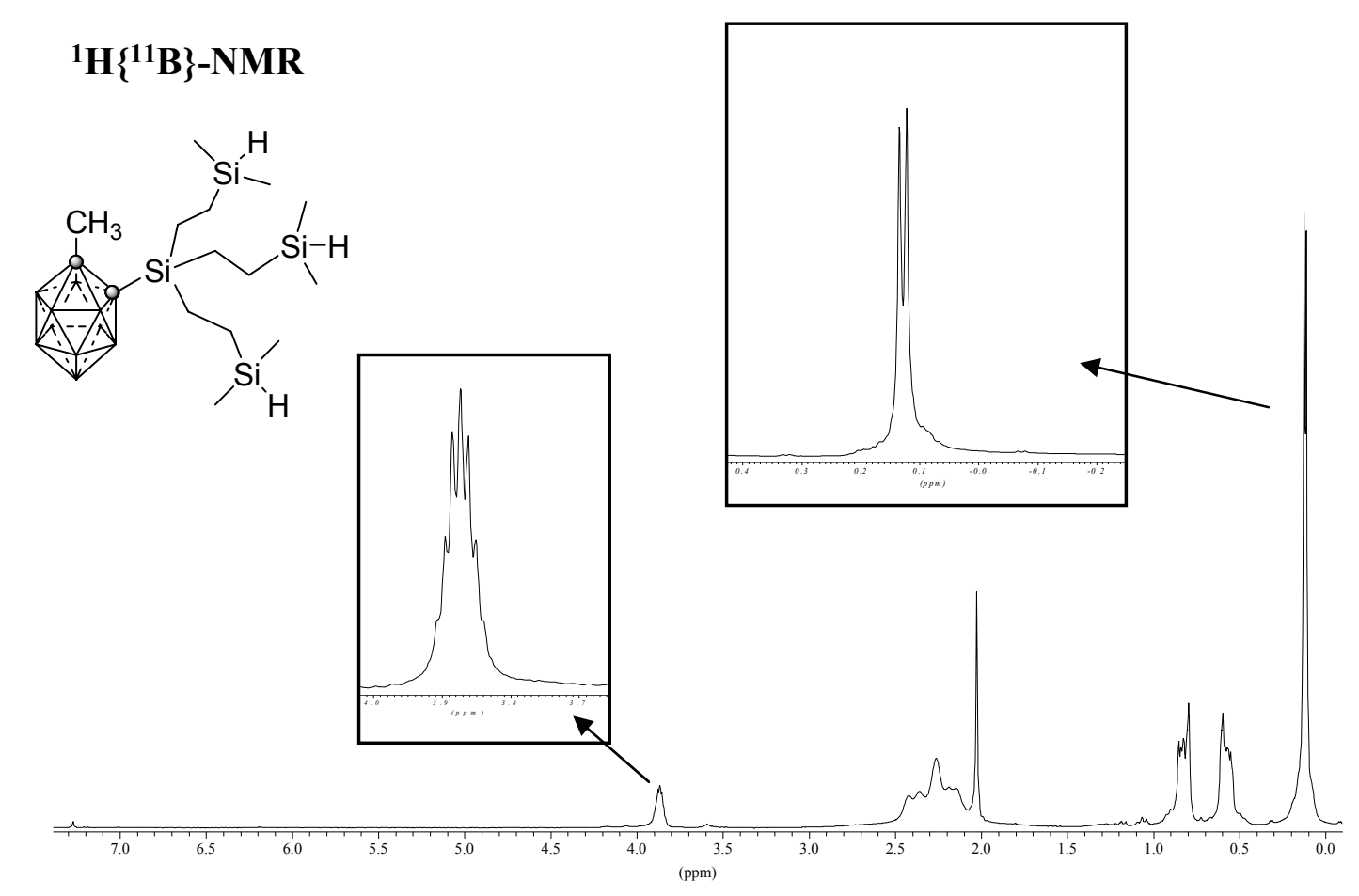

${ }^{13} \mathrm{C}\left\{{ }^{1} \mathrm{H}\right\}-\mathrm{NMR}$

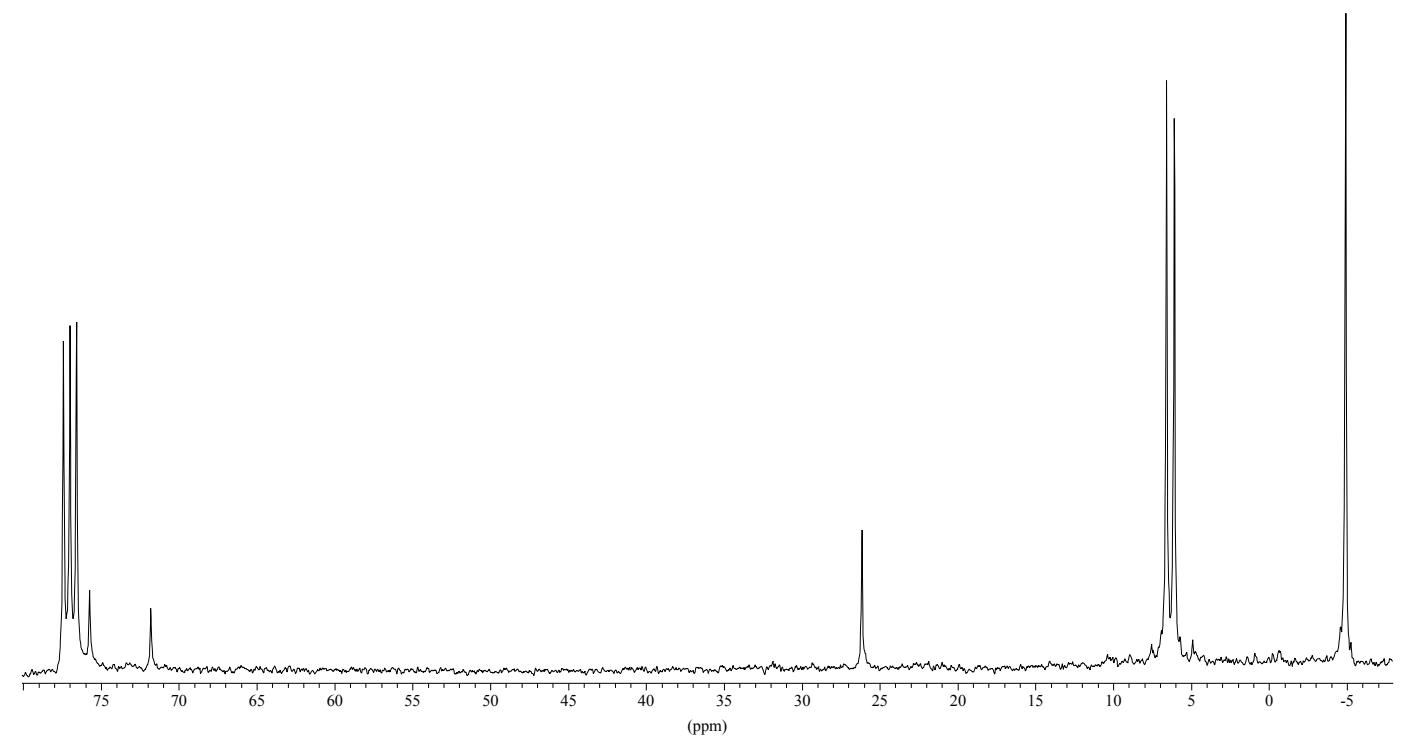


NMR Spectra of 9

\section{${ }^{1} \mathbf{H}\left\{{ }^{11} \mathbf{B}\right\}-\mathbf{N M R}$}
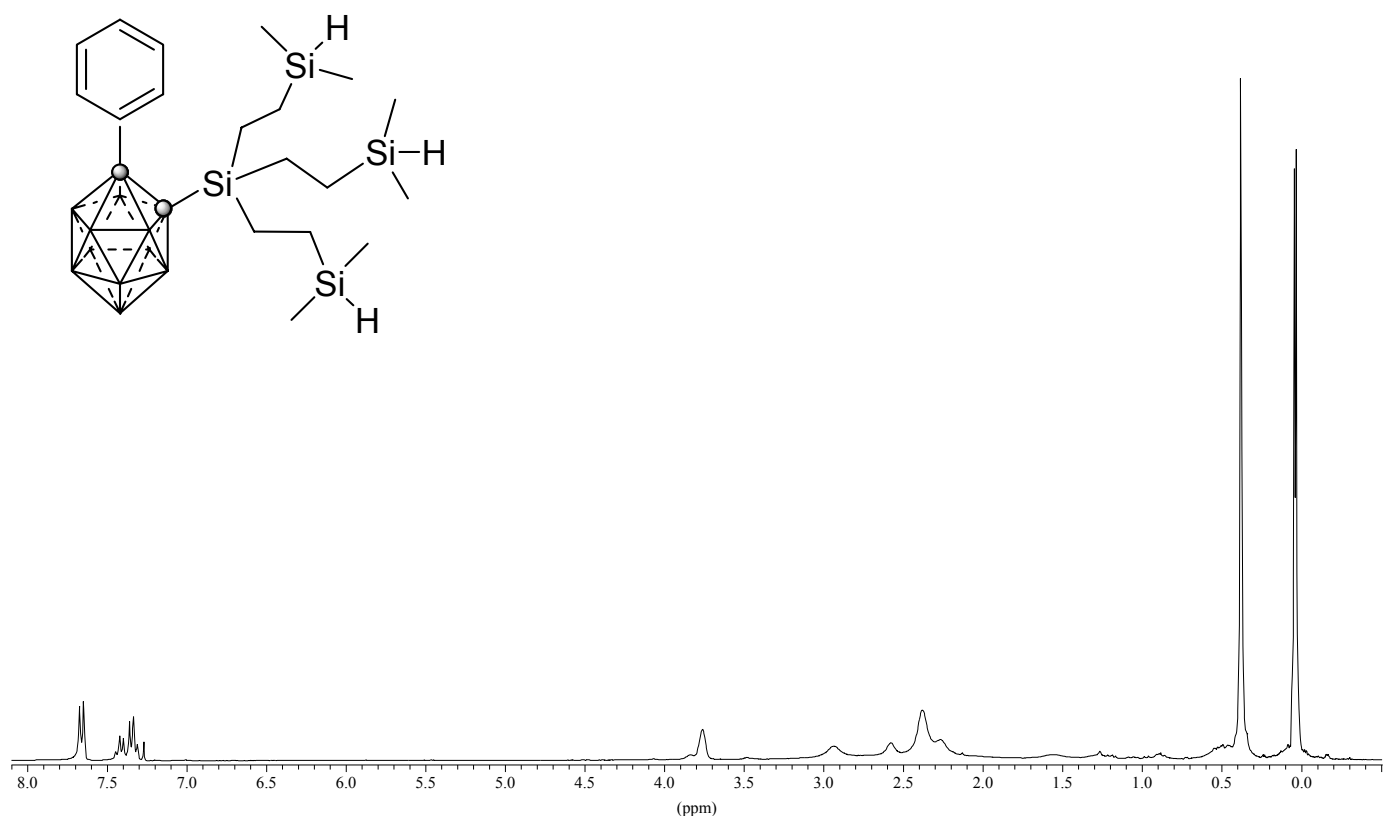

${ }^{13} \mathrm{C}\left\{{ }^{1} \mathrm{H}\right\}-\mathrm{NMR}$

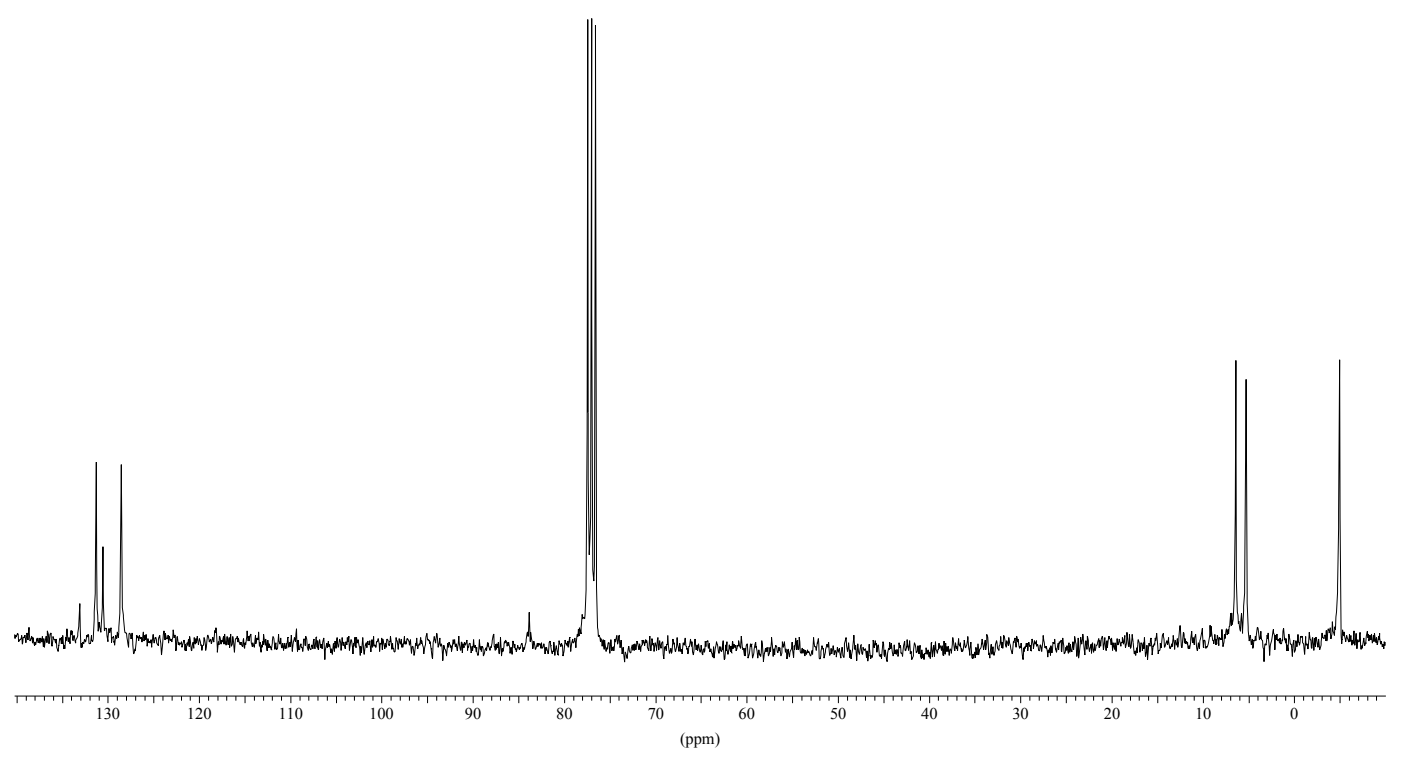


NMR Spectra of 10

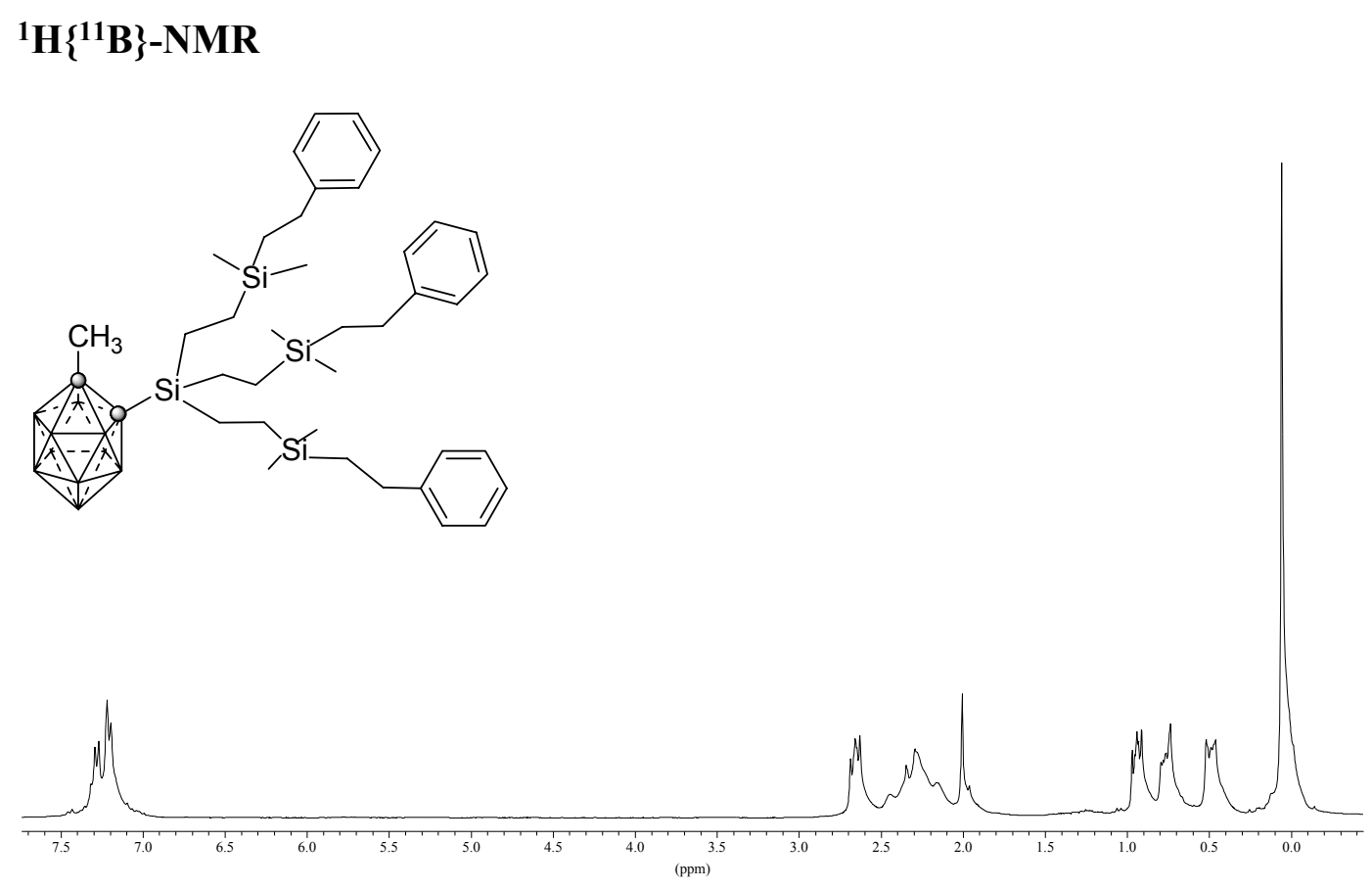

${ }^{13} \mathrm{C}\left\{{ }^{1} \mathrm{H}\right\}-\mathrm{NMR}$

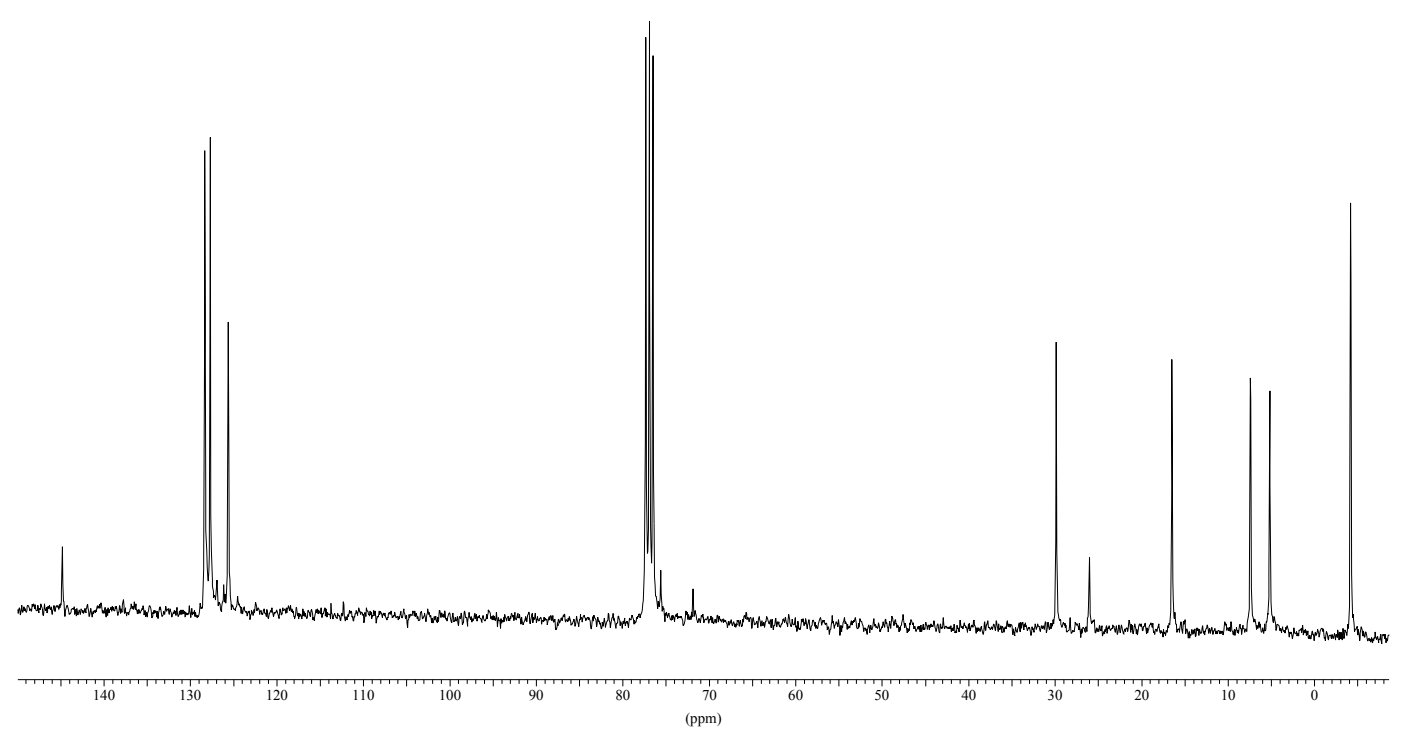




\section{NMR Spectra of 11}

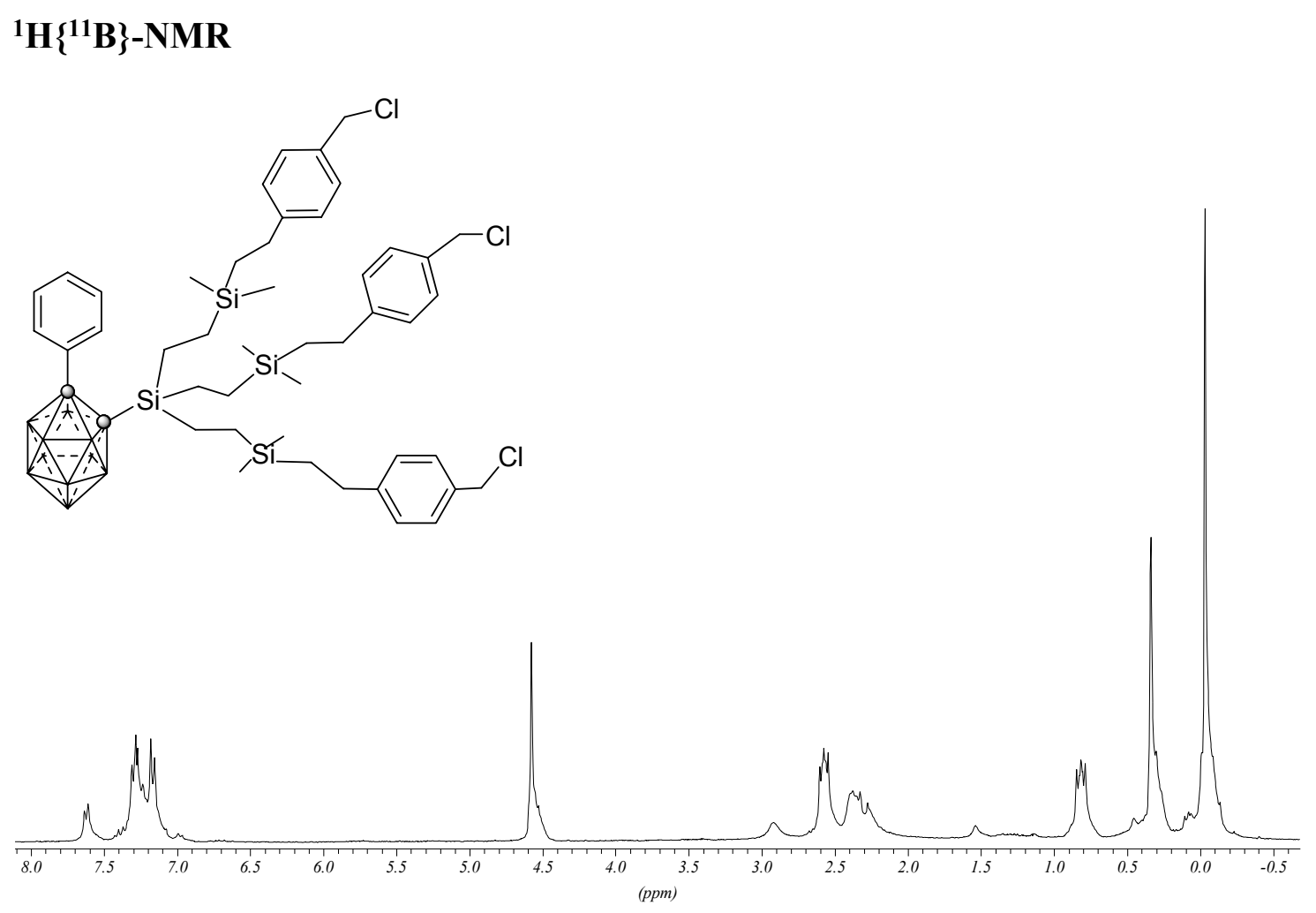

\section{${ }^{13} \mathrm{C}\left\{{ }^{1} \mathrm{H}\right\}-\mathrm{NMR}$}

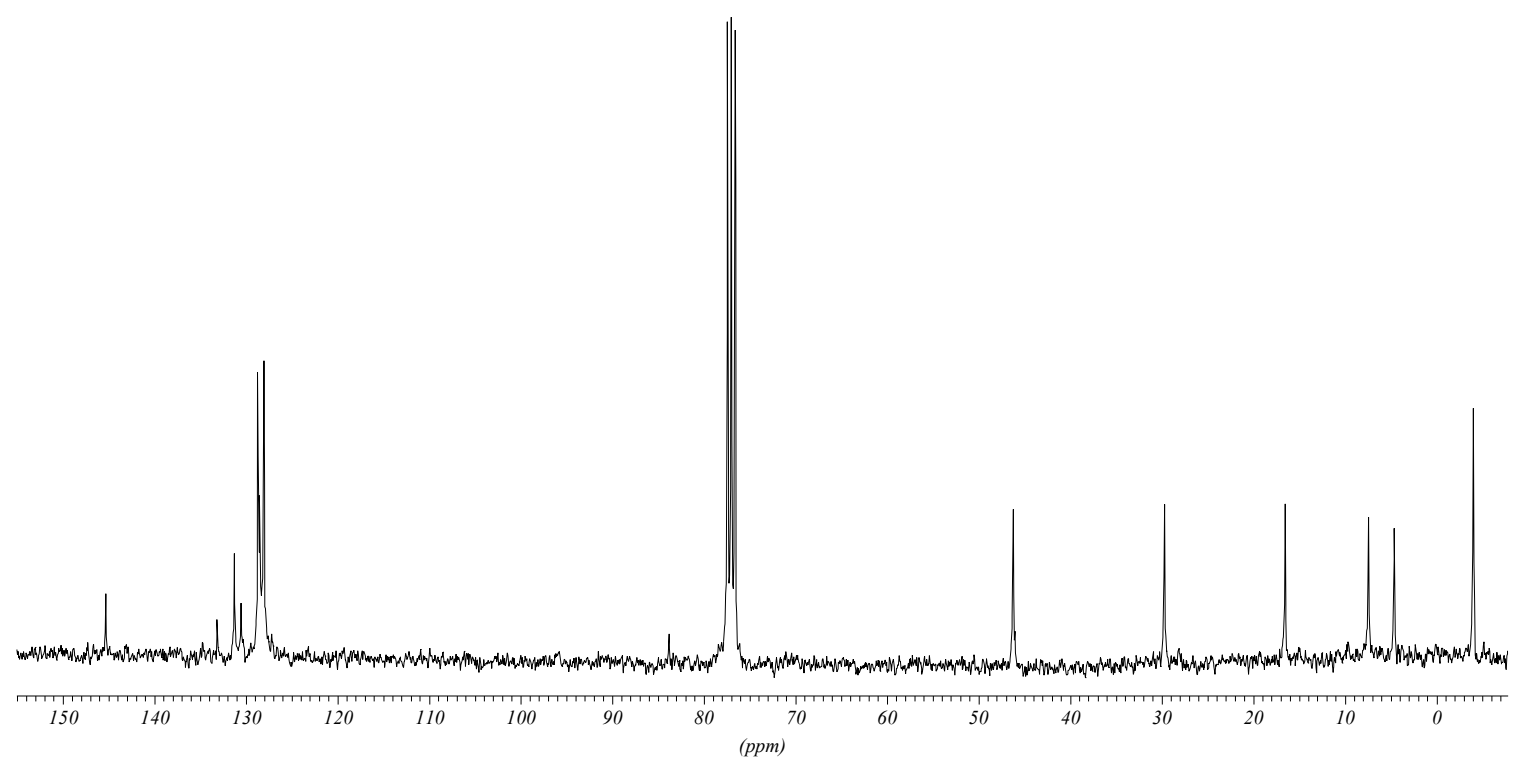




\section{NMR Spectra of 12}

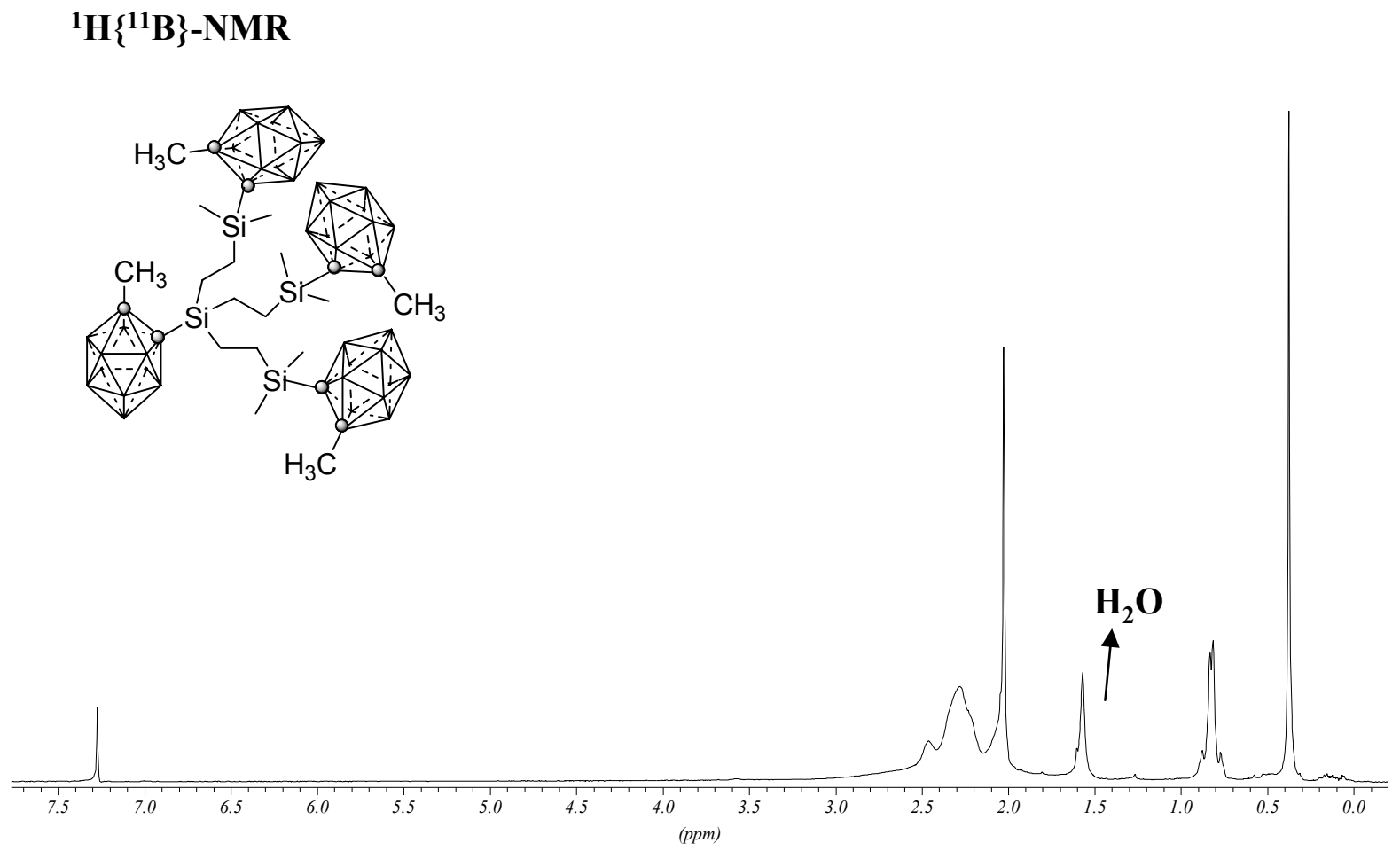

${ }^{13} \mathrm{C}\left\{{ }^{1} \mathrm{H}\right\}-\mathrm{NMR}$

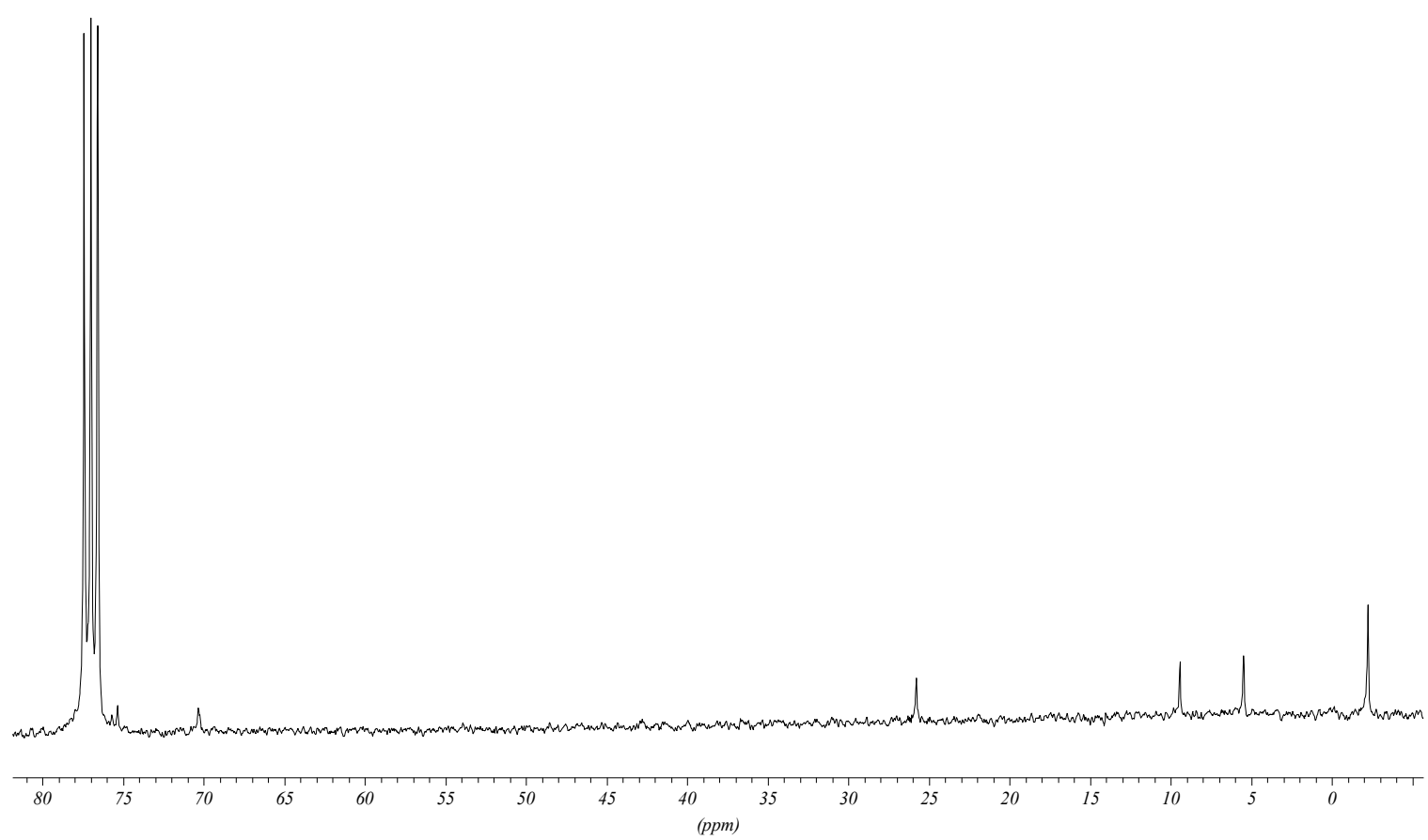




\section{NMR Spectra of 13}

\section{${ }^{1} \mathbf{H}\left\{{ }^{11} \mathbf{B}\right\}-\mathrm{NMR}$}
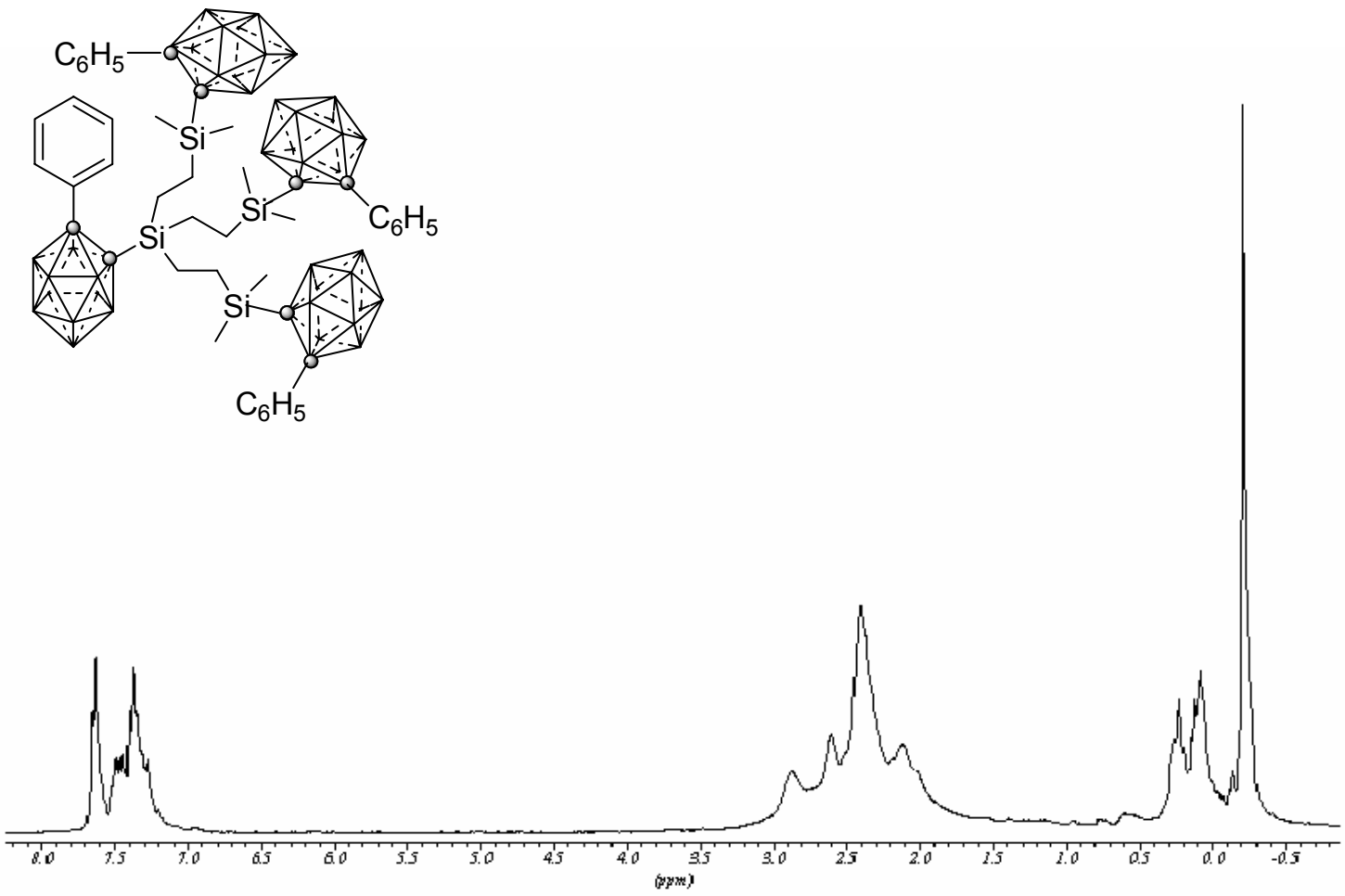

${ }^{13} \mathrm{C}\left\{{ }^{1} \mathrm{H}\right\}-\mathrm{NMR}$

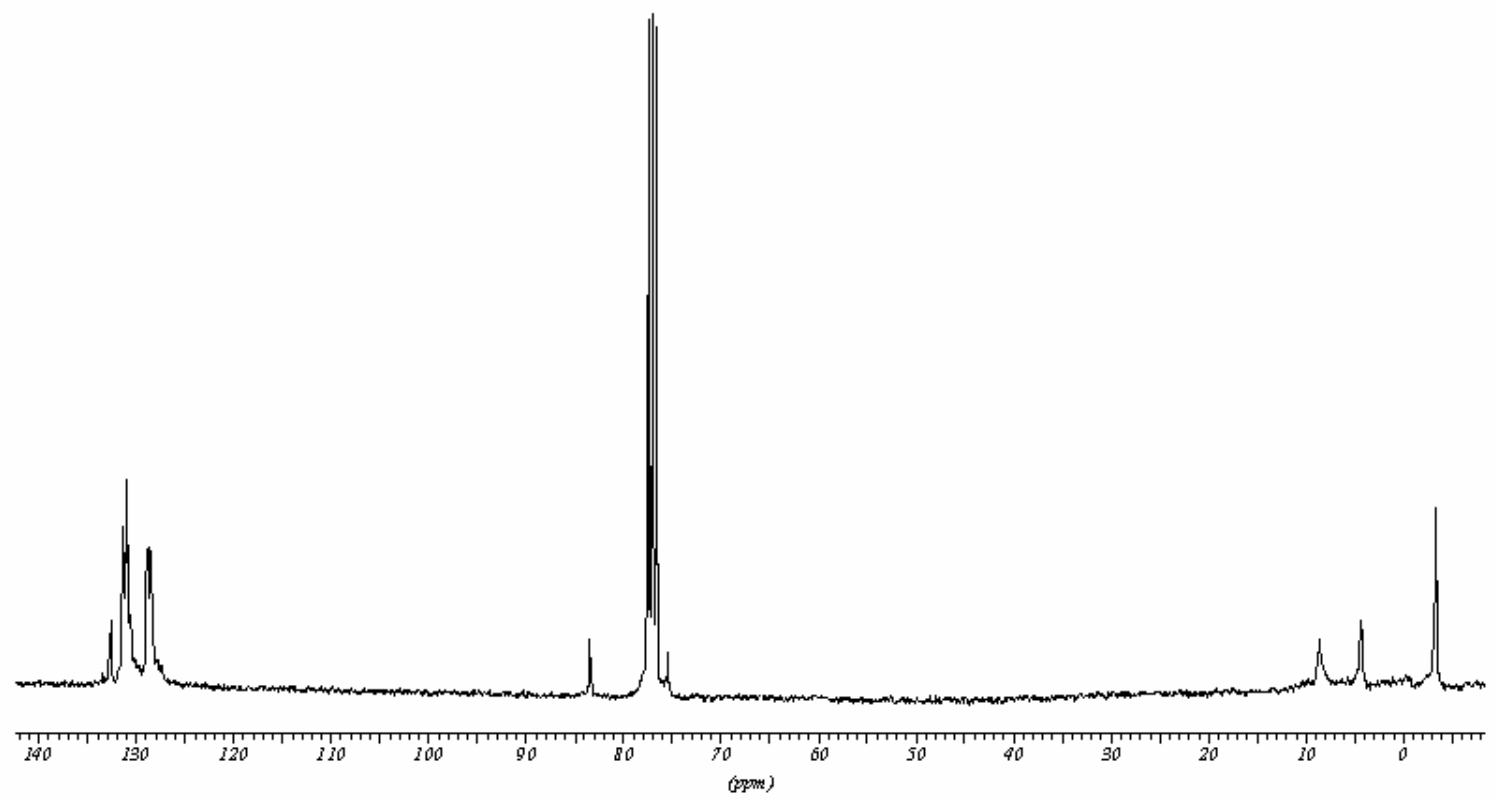




\section{NMR Spectra of 14}

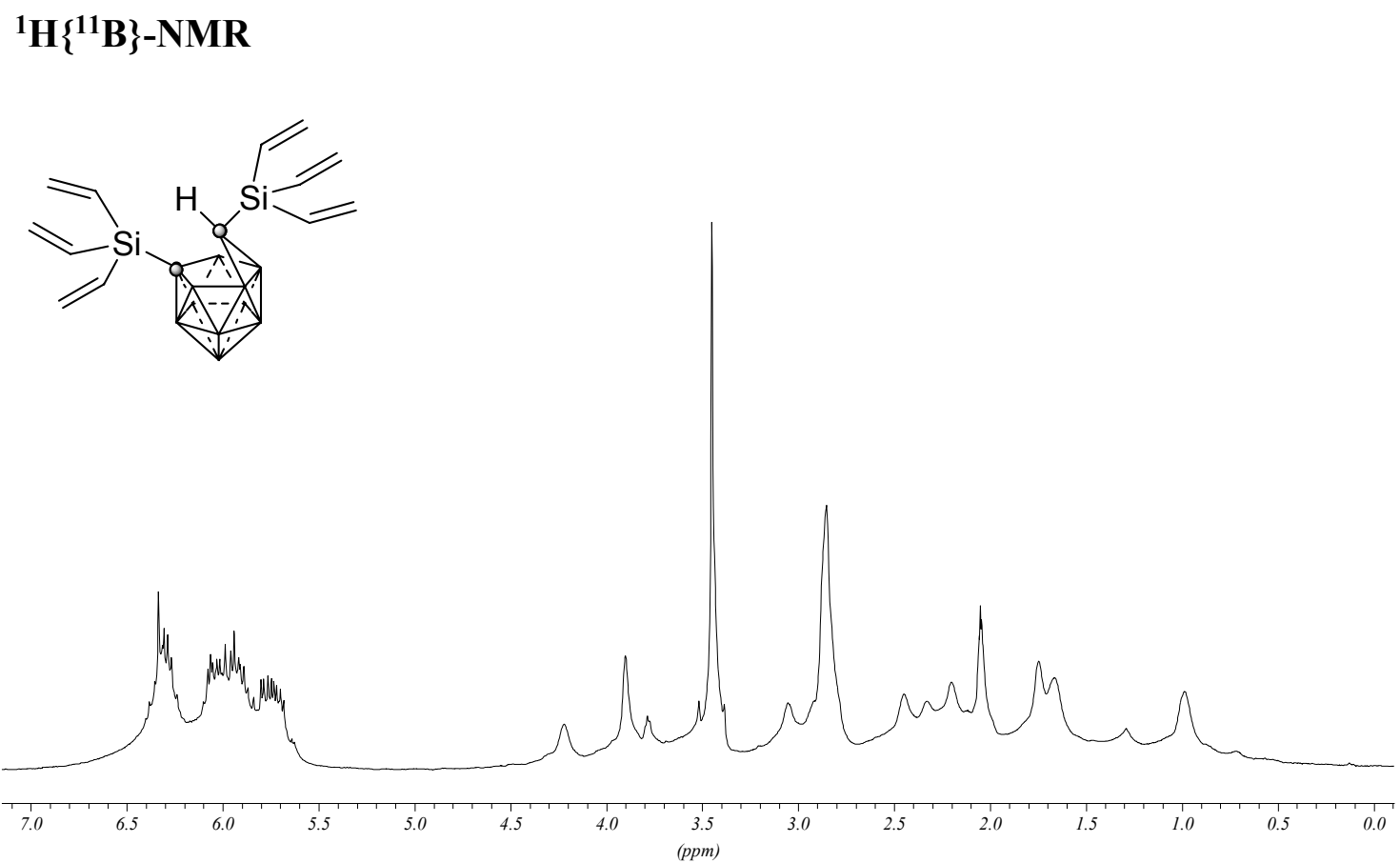

${ }^{13} \mathrm{C}\left\{{ }^{1} \mathrm{H}\right\}-N M R$

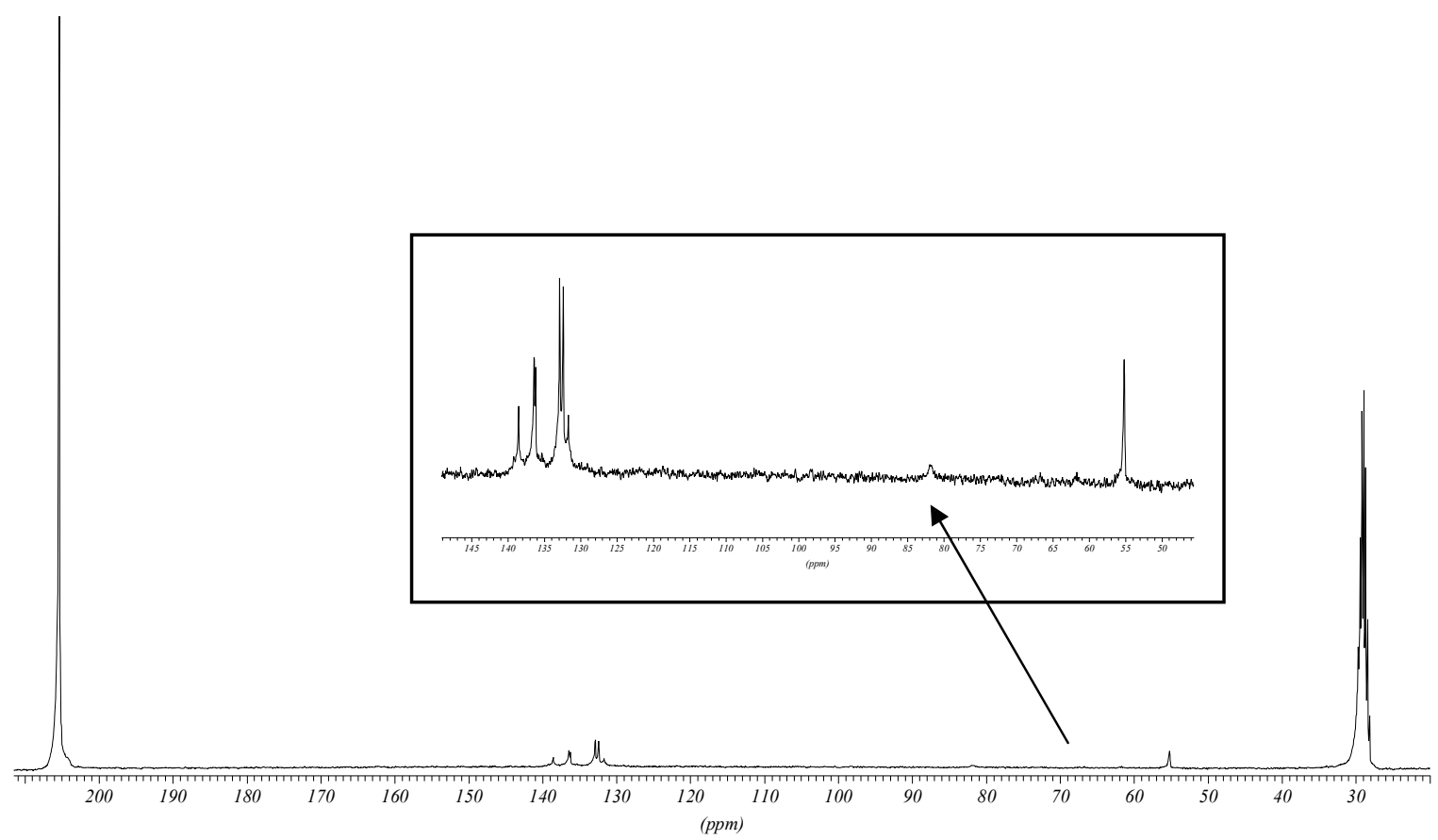




\section{NMR Spectra of 15}

${ }^{1} \mathrm{H}\left\{{ }^{11} \mathrm{~B}\right\}-\mathrm{NMR}$

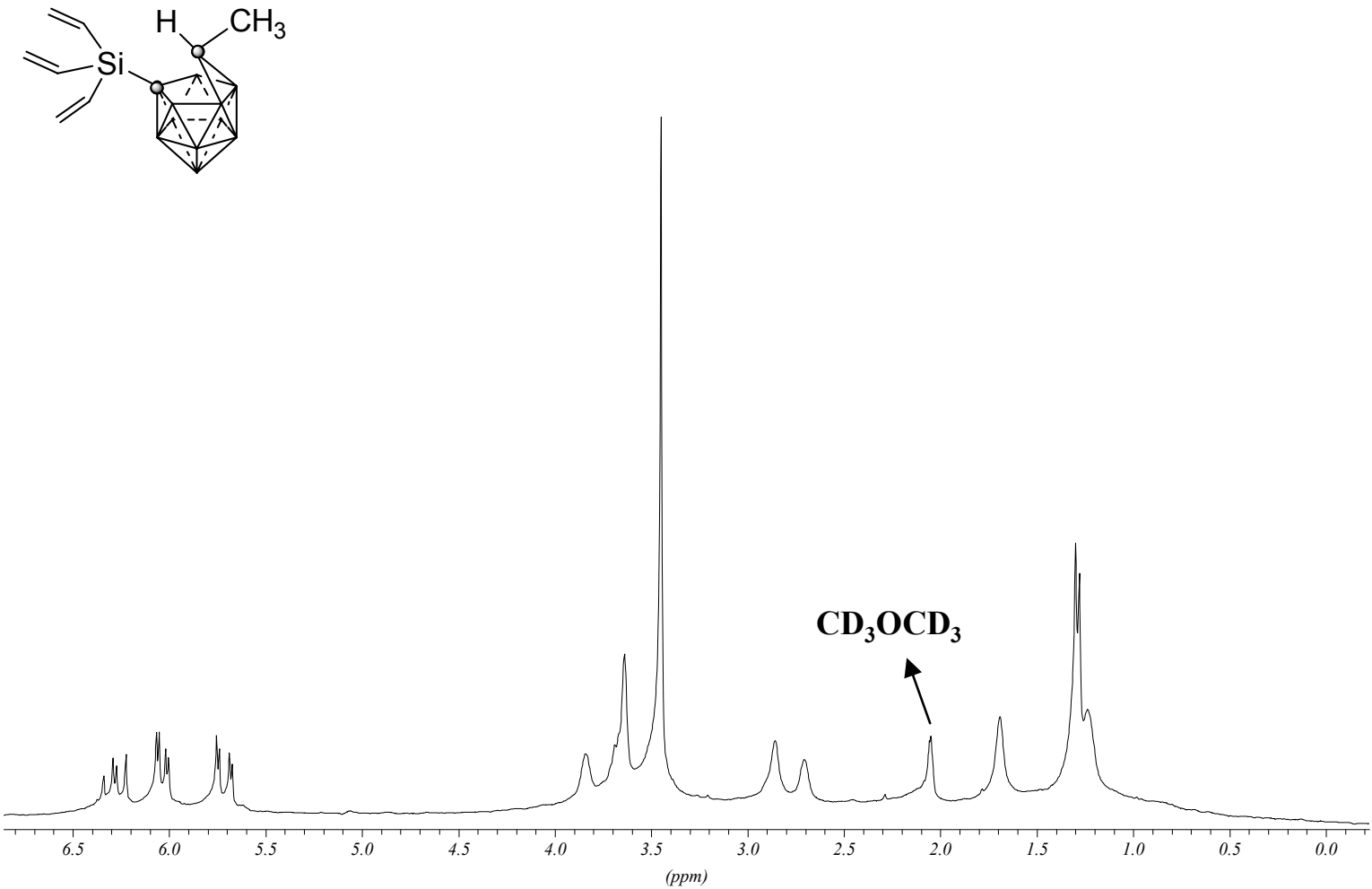

${ }^{13} \mathrm{C}\left\{{ }^{1} \mathrm{H}\right\}-\mathrm{NMR}$

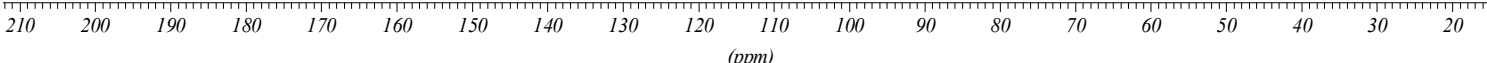


NMR Spectra of 16

${ }^{1} \mathrm{H}\left\{{ }^{11} \mathrm{~B}\right\}-\mathrm{NMR}$

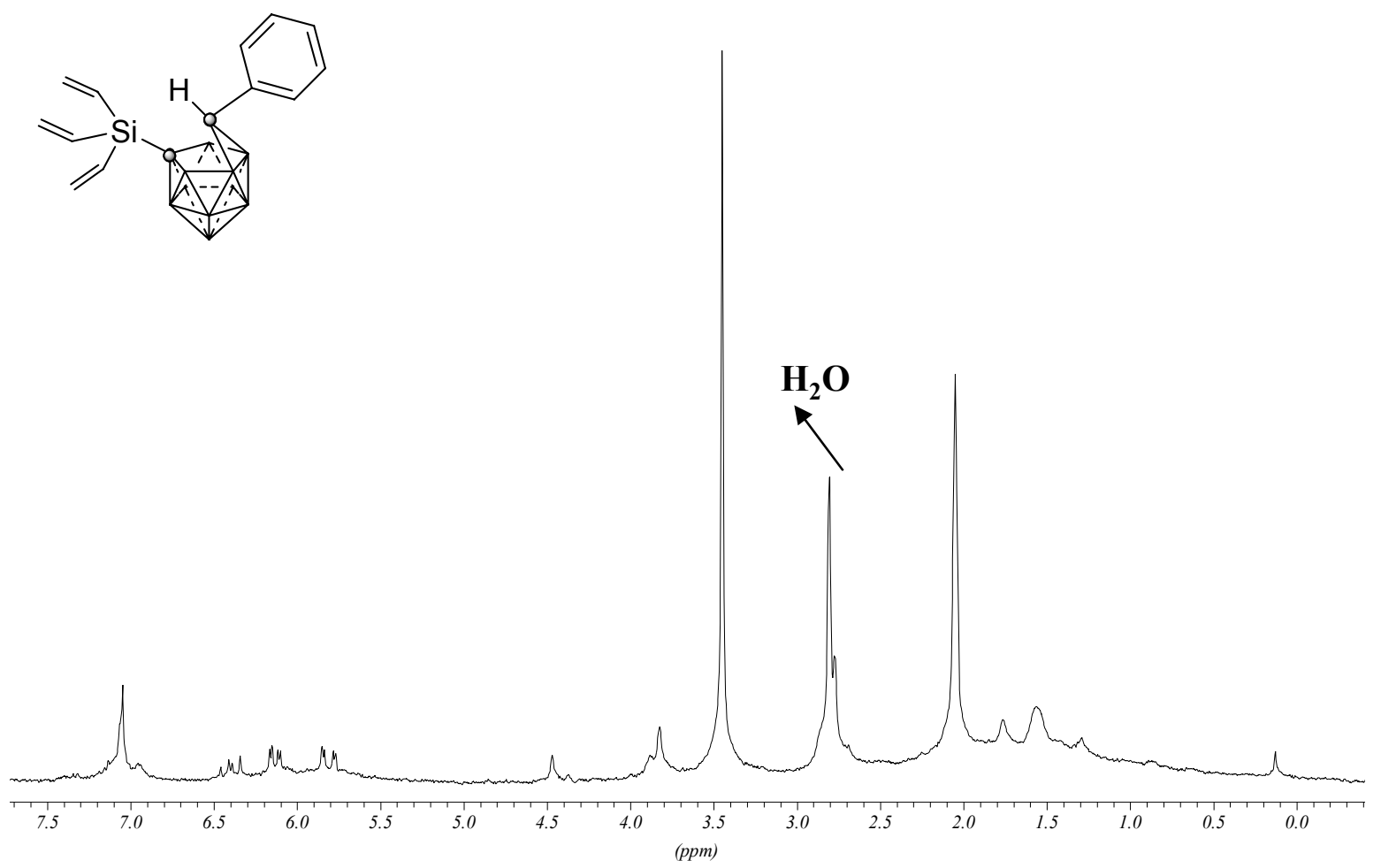

${ }^{13} \mathrm{C}\left\{{ }^{1} \mathrm{H}\right\}-\mathrm{NMR}$

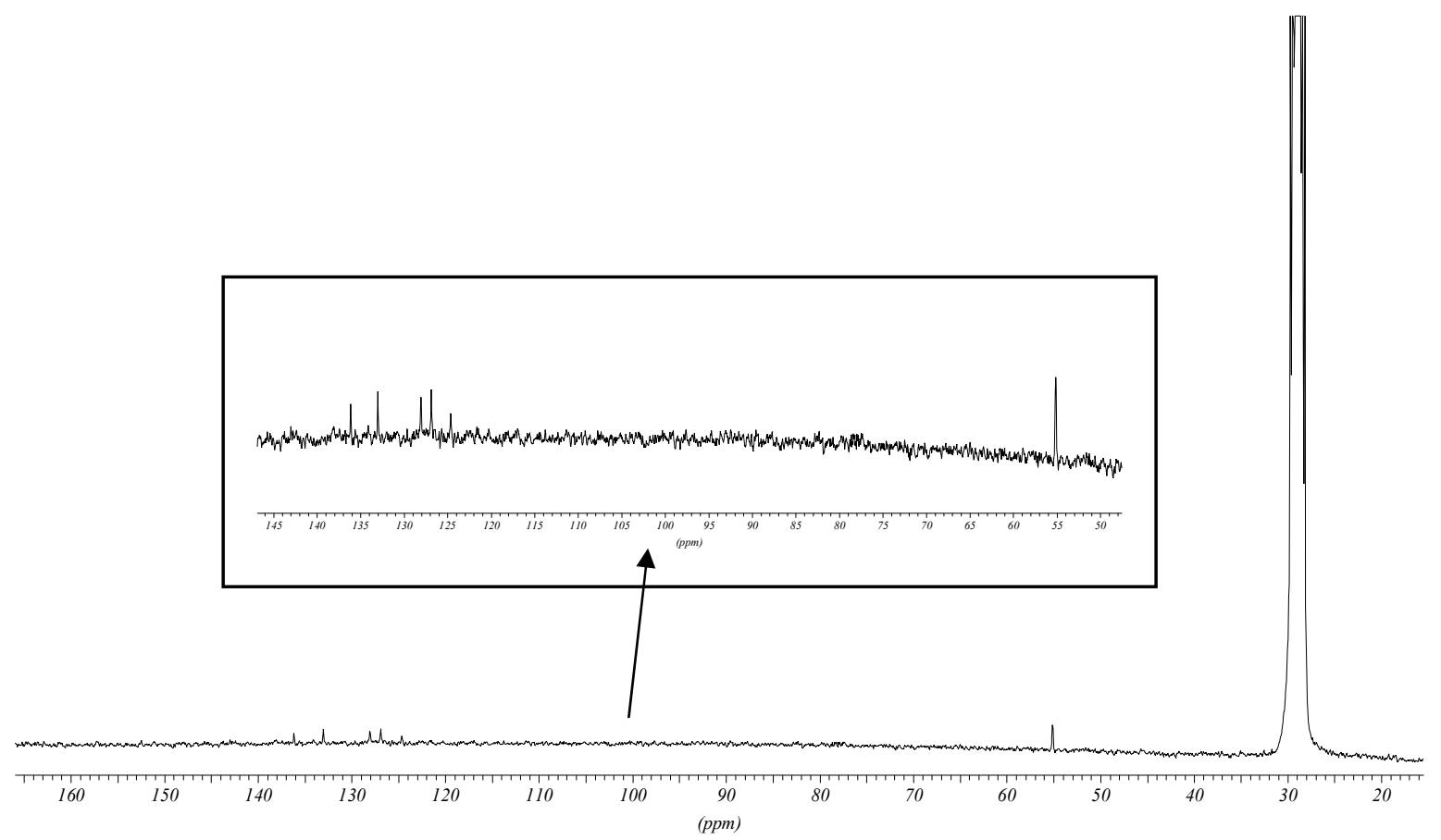


Table 1. Proton resonances on the ${ }^{1} \mathrm{H}$ NMR spectra of trivinylsilane groups

\begin{tabular}{cccc}
\hline Compound & $\mathrm{H}^{\mathrm{a}}$ & $\mathrm{H}^{\mathbf{b}}$ & $\mathrm{H}^{\mathbf{c}}$ \\
\hline $\mathbf{1 0}$ & $6.27-6.17$ & $6.27-6.17$ & 5.84 \\
$\mathbf{1 1}$ & 6.22 & 6.34 & 5.92 \\
$\mathbf{1 2}$ & 5.77 & 6.07 & 5.58 \\
$\mathbf{1 3}$ & $6.34-5.79$ & $6.34-5.79$ & $6.34-5.79$ \\
$\mathbf{1 4}$ & 6.28 & 6.03 & 5.71 \\
$\mathbf{1 5}$ & 6.39 & 6.13 & 5.81 \\
\hline
\end{tabular}


Table 2.- Symmary of Crystallographic Data for 1-3 at 173(2) K

\begin{tabular}{|c|c|c|c|}
\hline & 1 & 2 & 3 \\
\hline empirical formula & $\mathrm{C}_{14} \mathrm{H}_{28} \mathrm{~B}_{10} \mathrm{Si}_{2}$ & $\mathrm{C}_{9} \mathrm{H}_{22} \mathrm{~B}_{10} \mathrm{Si}$ & $\mathrm{C}_{14} \mathrm{H}_{24} \mathrm{~B}_{10} \mathrm{Si}$ \\
\hline formula weight & 360.64 & 266.46 & 328.52 \\
\hline crystal habit, color & prism, colorless & block, colorless & prism, colorless \\
\hline crystal system & monoclinic & triclinic & monoclinic \\
\hline space group & $P 2_{1} / c$ (no. 14$)$ & $P-1$ (no. 2) & $P 2_{1} / c$ (no. 14 ) \\
\hline$a(\AA)$ & $15.8018(8)$ & $7.7775(2)$ & $16.7308(12)$ \\
\hline$b(\AA)$ & $10.5930(6)$ & $14.1714(3)$ & $13.0118(5)$ \\
\hline$c(\AA)$ & $13.4179(4)$ & $15.9281(5)$ & $19.7733(13)$ \\
\hline$\alpha(\operatorname{deg})$ & 90 & $96.034(2)$ & 90 \\
\hline$\beta(\operatorname{deg})$ & $99.317(3)$ & $103.042(2)$ & $113.094(2)$ \\
\hline$\gamma(\operatorname{deg})$ & 90 & $100.443(2)$ & 90 \\
\hline$V\left(\mathrm{~V}^{3}\right)$ & $2216.37(18)$ & $1662.30(8)$ & $3959.6(4)$ \\
\hline$Z$ & 4 & 4 & 8 \\
\hline$\rho\left(\mathrm{g} \mathrm{cm}^{-3}\right)$ & 1.081 & 1.065 & 1.102 \\
\hline$\mu\left(\mathrm{cm}^{-1}\right)$ & 1.56 & 1.19 & 1.12 \\
\hline no. measured reflns. & 7421 & 10437 & 13072 \\
\hline$R_{\text {int }}$ & 0.0466 & 0.0262 & 0.0414 \\
\hline goodness-of-fit ${ }^{\mathrm{a}}$ on $F^{2}$ & 1.046 & 1.030 & 1.041 \\
\hline$R^{\mathrm{b}}[I>2 \sigma(I)]$ & 0.0460 & 0.0426 & 0.0535 \\
\hline$R_{w}{ }^{\mathrm{c}}[I>2 \sigma(I)]$ & 0.1055 & 0.1070 & 0.1230 \\
\hline
\end{tabular}

